Meta

Journal des traducteurs

Translators' Journal

\title{
Quelques facteurs sociodémographiques qui sous-tendent le choix des langues pour la terminologie en milieu de travail et leur interrelation avec des attitudes
}

\section{Denise Daoust}

Volume 40, numéro 2, juin 1995

Usages sociaux des termes : théories et terrains

URI : https://id.erudit.org/iderudit/002458ar

DOI : https://doi.org/10.7202/002458ar

Aller au sommaire du numéro

\section{Éditeur(s)}

Les Presses de l'Université de Montréal

\section{ISSN}

0026-0452 (imprimé)

1492-1421 (numérique)

\section{Découvrir la revue}

\section{Citer cet article}

Daoust, D. (1995). Quelques facteurs sociodémographiques qui sous-tendent le choix des langues pour la terminologie en milieu de travail et leur interrelation avec des attitudes. Meta, 40(2), 260-283. https://doi.org/10.7202/002458ar
Résumé de l'article

Une série d'édudes d'opinions réalisées en 1983 et en 1990 dans une grande entreprise, à Montréal, révèle qu'il existe un lien entre le choix déclaré du français ou de l'anglais pour un ensemble de 10 termes techniques testés, et certains facteurs sociodémographiques et occupationnels. Les analyses statistiques ont démontré qu'en règle générale, les femmes, les travailleurs plus instruits, de même que ceux qui occupent des postes de gestion et de col blanc déclarent une plus grande utilisation du français pour les termes testés. Il s'avère aussi que ces mêmes catégories de travailleurs ont tendance à exposer des opinions et à montrer des comportements révélateurs d'attitudes plus favorables au français et au processus de francisation terminologique de leur entreprise que les autres travailleurs. Cependant, les facteurs extralinguistiques examinés ne jouent pas tous un rôle d'égale importance. Des analyses statistiques de régression ont permis de hiérarchiser ces facteurs, et de montrer ainsi le rôle primordial des opinions et comportements révélateurs d'attitudes. Il devient donc possible d'établir le portrait type du travailleur susceptible de privilégier la terminologie de langue française et, sans doule, de modifier ses habitudes terminologiques vers une plus grande utilisation du français. Cependant, les liens qu'entretiennent entre elles chacune des variables étudiées sont très complexes, et leur étude mène à un questionnement théorique et méthodologique par rapport aux facteurs qui sous-tendent l'usage des langues el à l'interprétation de la dynamique sociolinguistique qui résulte de leur interrelation, de même que par rapport à la relation qui existe entre le changement linguistique qui s'opère naturellement et le changement planifié qui s'inscrit dans un contexte d'aménagement linguistique.
Tous droits réservés @ Les Presses de l'Université de Montréal, 1995
Ce document est protégé par la loi sur le droit d'auteur. L'utilisation des services d'Érudit (y compris la reproduction) est assujettie à sa politique d'utilisation que vous pouvez consulter en ligne.

https://apropos.erudit.org/fr/usagers/politique-dutilisation/ 


\title{
QUELQUES FACTEURS SOCIODÉMOGRAPHIQUES QUI SOUS-TENDENT LE CHOIX DES LANGUES POUR LA TERMINOLOGIE EN MILIEU DE TRAVAIL ET LEUR INTERRELATION AVEC DES ATTITUDES'
}

\author{
DHANIS: DAOUST

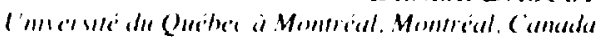

\begin{abstract}
Résumé

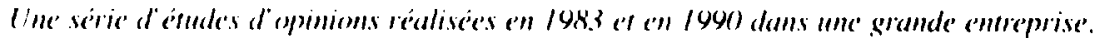

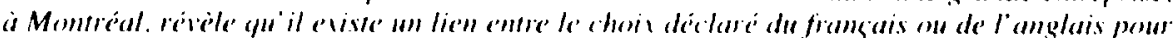

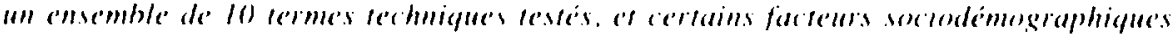

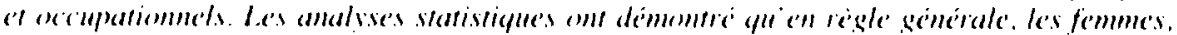

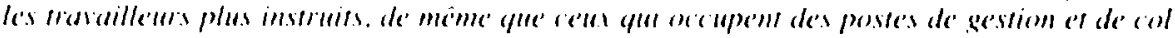

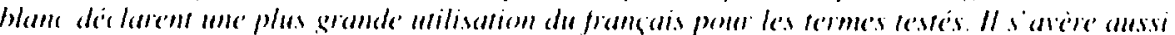

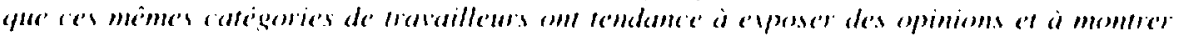

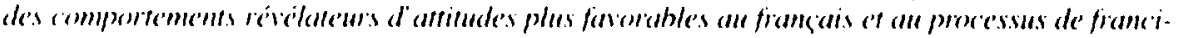

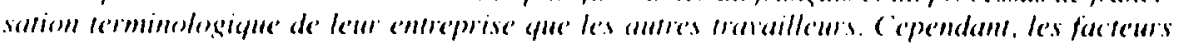

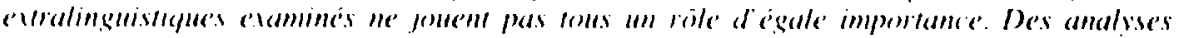

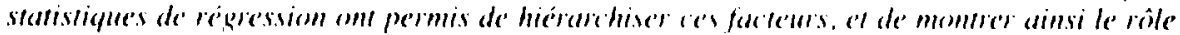

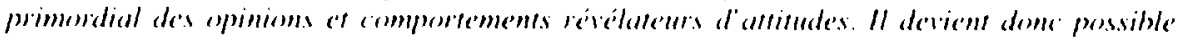

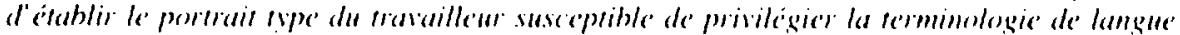

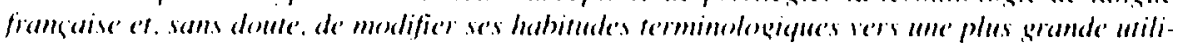

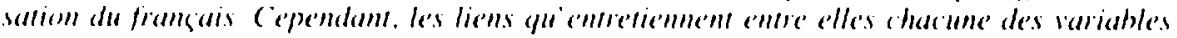

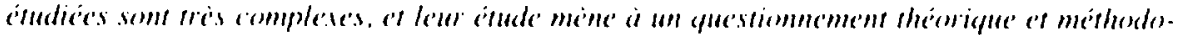

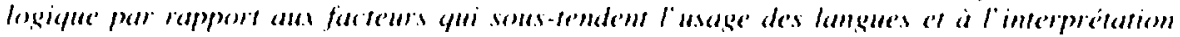

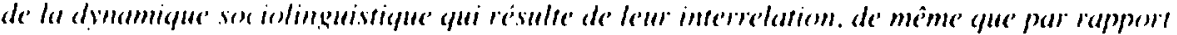

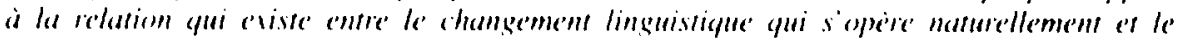

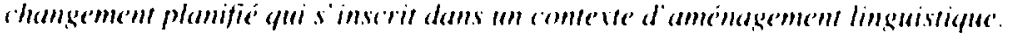

\section{INTRODUCTION}

L'élude de la situation linguistique en milieu de travail au Québec, où le français et l'anglais sont en concurrence, soulève de nombreuses questions sur la hiérarchisation sociolinguistique des langues et sur la notion même de communauté linguistique. Ainsi. la "Charte de la langue française", la loi linguistique quétrécoise, en vigueur depuis août 1977. déclare le français langue officielle du Québec, ainsi que langue des domaines publics. des affaires et du monde du travail.

Théoriquement donc. le français. de par son statut politique, jouit, au Québec, d'un prestige social important. Mais qu en est-il de son statut sociolinguistique réel? Fn fait. pour comprendre celte question, il faut tenir compte de la complexité de la situation québécoise, où la concurrence linguistique s'exerce non seulement entre deux langues distinctes, 
le français et l'anglais. mais aussi entre deux variétés linguistiques du français: le français hexagonal et le français québécois. Par ailleurs, la situation est compliquée par le fait que le français et l'anglais sont aussi en concurrence sur le plan international. Au Québec. donc, au prestige de l'anglais, langue internationale de la science, de la technologie et des affaires, s'ajoute celui du français hexagonal qui tend a s"imposer dans la francophonie. Les normes sociolinguistiques de la communauté québécoise sont donc extrêmement complexes puisqu'elles font appel simultanément à des normes "locales» d'utilisation linguistique et sociolinguistique du français et de l'anglais parlés au Québec, et à des normes supra-dialectales, voire internationales, qui gèrent l'usage et le statut de ces deux langues de prestige. Pour ce qui est du monde du travail, l'anglais y est, comme un peu partout dans le monde. largement utilisé pour les termes techniques: il s'avère que même les travailleurs dont la langue première est le français sont soumis aux pressions de l'anglais, et bon nombre d'entre eux utilisent une terminologie partiellement anglaise. De plus. l'entreprise québécoise connaît un autre type de concurrence terminologique, entre les termes dits du français québécois, el ceux généralement préconisés par l'Office de la langue française'2 qui s'alignent souvent sur la norme du français hexagonal.

Il s'ensuit que ces différentes normes, souvent conflictuelles, créent une situation où l'emprunt linguistique se situe à plusieurs niveaux. Dans un tel contexte. il y a forcément chevauchement entre la variation linguistique, entendue dans le sens d'utilisation de variantes linguistiques d'une même langue (Labov 1966). et l'emprunt, qui réfère à l'utilisation de structures linguistiques d'une langue différente, ces structures étant plus ou moins intégrées à la langue emprunteuse. Ceci est particulièrement évident dans le cas d'emprunts de mots isolés ou d'expressions lexicalisées, de sorte qu'il s'avère justifié. dans certains contextes du moins, de considérer l'ensemble de ces phénomènes comme faisant partie d'un continuum (Fasold 1984: 183)?

Cette approche n'est d'ailleurs pas incompatible avec la conception de la sociolinguistique variationniste, qui considère que l'utilisation de variantes linguistiquement synonymiques mais socialement pertinentes révèle les règles sociolinguistiques qui sous-tendent l'utilisation des langues et définissent. par le fait même. le modèle de la astratification sociale" d'une communauté linguistique (Labov 1966, 1969 et 1972b: 121). D'ailleurs. Labov, le principal représentant de cette école, adopte un critère sociolinguislique pour définir la communauté linguistique. Celle-ci est caractérisée, selon lui, non pas lant par un consensus sur l'utilisation de structures linguistiques, mais surtout par ala participation à un ensemble de normes communes», que l'on peut inférer à la fois des jugements ou ucomportements évaluatifs" concernant la langue, et des "schémas abstraits de la variation". cest-à-dire les structures linguistiques et sociolinguistiques qui soustendent le phénomène de la variation linguistique dans un milieu donné (ma traduction. Labov 1972h: 120-121). C"est donc le compontement linguistique en situation réelle couplé à la perception des normes linguistiques et sociolinguistiques en cours dans la société qui définit la communauté linguistique ${ }^{t}$.

Enfin. lélude de la situation sociolinguistique du monde du travail québécois s'avère intéressante à un autre point de vue. Élant donné que la "Charte de la langue française" (dite. "loi 101") oblige l'entreprise à diffuser et à assurer l'utilisation d'une terminologie de langue françaixs". le milieu de l'entreprise dans lequel le changement vers la francisation doit sopérer constitue. en quelque sorte, un contexte contrôlé. une sorte de laboratoire du changement linguistique et sociolinguistique qu' on peut qualifier de "planifié", par opposition au changement "naturel" qui seffectue dans un contexte de libre concurrence entre les divers facteurs sociaux. économiques, politiques et culturels qui composent toute communauté linguistique. 


\section{Le changement naturel et le changement planifié}

On connaît assez bien le rôle des facteurs sociodémographiques, socio-économiques et psychosociaux yui conditionnent l'utilisation des langues et le changement linguistique. Par exemple, la sociolinguistique nous apprend que l'utilisation d'une variante normative est habituellement en corrélation avec un niveau socio-économique élevé ou un niveau de scolarité élevé. On sait aussi que les femmes. de même que les groupes plus âgés, utilisent davantage les formes normatives dans un contexte de variation yu on pourrait qualifier de stable (Chambers et Trudgill 1980: 67-100): Labov 1966, 1972b, 1981 et 199(): Milroy 1980 el 1987: Wolfram 1969). Par contre, les femmes sont souvent i l'avant-garde du changement dans un contexte où une variante lend à être perçue comme socialement valorisée (Chambers et Trudgill 1980: 97-98: Labov 1981: 18.5 et 1990). Quant au facteur age bien qu il y ait des exceptions, ce sont les jeunes qui utilisent davantage les varianles novatrices (Chambers et Trudgill 1980: 91-94: Labov 1966). De même, certaines variantes non valorisées sont associées à des groupes sociaux, souvent minoritaires, socio-économiquement ou culturellement parlant. dont la variété linguistique est dite domince ou minoréc (Trudgill 1979: 19 et 1983: 109-110). Enfin, il ressort que cest la classe moyenne qui est habituellement a lorigine du changement linguistique (Labov 1972b: Trudgill 1974)". (ependant, il appert que, dans certains contextes, la différenciattion socio-économique est subordonnée. en quelque sorte, à une différenciation qui prend en comper la relation socio-economique et culturelle qu entretiennent certans individus avec la langue standard, ou "légitime" (Bourdieu el Boltanski 1975). Dans ce contexte. certaines catégories d'individus. tels les enseignants. les avocalts, les préposés a l'accueil, etc., sont classées selon une échelle qui prend en compte le fait que leurs fonctions, qu'on pourrait qualifier de epubliques». maximisent l'utilisattion d'une variété linguistique plus prestigieuse. et habituellement normative (Sankoff el Laberge 1978: 239-250). Par ailleurs, la sociolinguistique distingue deux catégories de changement linguistique. selon qu il s'agit d'un changement apparemment inconscient (afiom bolow |the level of awareness]" (L abov 1966). et dont on pourrail retracer l'origine. dans certains cas. chez les locuteurs de lat classe ouvriere (Labov 1966). ou d'un changement conscient

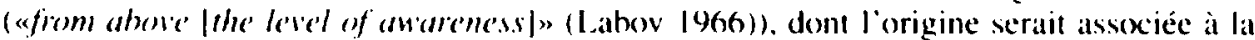
classe sociale moyenne (Labov 1966)?

Cependant. on connaît bien moins le rôle que ces mêmes facteurs jouent dans le contexte du changement planitié. e'est-a-dire du changement qui s'inscrit dans une politique d'aménagement d'une langue. Ljune des caractéristiques les plus importantes de ce type de changement réside dams le fait que le systène de départ est connu. tout comme le système d'arrivée, ou tout alu moins. le systeme idéal visé. Fi. même si lè changement visé respecte les tendances naturelles de la langue qu on veut modifier. ou orienter, il suppose, à tout le moins, une accélération du processus naturel. Parfois, cependant, on tente d'inverser les lendances de l'évolution naturelle. Par ailleurs, dans certains contextes. comme au Québec. la période d'implantation. durant laquelle le changement doit s'effectuer. est délimitéc. Entin, du fait que le changement visé est un acte délibéré, on peut s"atlendre a ce que le niveau de conscience linguistique et sociolinguistique ches les groupes touches par ces politiques soit beaucoup plus élevé que dans les cas de changement naturel. De même. compte tenu du fail que lat cause du changement est extérieure aux locuteurs et nla pas comme siege principal le systeme proprement linguistique, et que kes groupes visés sont clairement identifies. il est sans doute possible de cerner. peut-être avec plus de certitude que dans le cas du changement naturel, les facteurs extralinguistiques yui jouent un rôle détenminant dans le processus d'utilisation des langues et du changement linguistique. 
L.FUDF: I.FS FACTEURS EXTRALINGOUISTIQUES RELIES AL CHOIX DES I.ANGUES POUR L.A TERMINOLCGIF, ET AI CHANGHMENT DU COMPORTEMENT TERMINOLOCIQUE: F.T SOCIOLINC;IISTIQUE:

\section{Description de l'étude}

C"est dans ce contexte général que s"inscrit la recherche. ou plutôt le programme de recherche présenté ici. qui vise, dans un premier temps, à identifier et à analyser certains des facteurs extralinguistiques qui sous-tendent le choix des langues pour la terminologie (de l'anglais ou du français) en milieu de travail. et dans un deuxième temps, à identifier et à analyser le rôle que ces mêmes facteurs jouent dans le processus de diffusion èt d'implantation d'une terminologic de langue française. A long terme. cefle recherche devrait permettre de mieux comprendre la dynamique du changement linguistique/ terminologique et sociolinguistique planifiék.

Pour ce faire, jai réalisé en 198.3. dans une grande entreprise de transponts de la région montréalaise. une enquête de type sociologique à l'aide d'un questionnaire d'opinions. écrit. auto-administré, d'une durée denviron vingt minutes, comprenant principalement des questions de type fermé. à choix multiples. Cette étude a permis de faire une première description et analyse de la situation sociolinguistique et terminologique dans celte entreprise. Dans un deuxième temps, en 1990 , j’ai recueilli des données comparables dans la même entreprise. afin de comparer la situation terminologique et sociolinguistique. et d'identifier les ficteurs reliés au processus du changement terminologique et sociolinguistique. Chacun des questionnaires (de 1983 et de 1990 ) a permis de recueillir. en moyenne. 150 variables pour chacun des répondants. L'échantillon fïnal de 1983 comprend 453 travailleurs de tous niveaux hiérarchiques $(60 \%$ des employés); celui de 1990 compte 192 répondants $(40 \%$ de la population totale à cetté époque). Ce deuxième corpus comprend deux cattégories de travailleurs: ceux qui travaillaient déjà en 198.3 lors de la première cueillette de données et que je désigne sous le nom des travailleurs du groupe $A$ (99 travailleurs). el ceux qui se sont ajoutés par la suite". Entīn, je suis retournéc une troisième fois dans la même entreprise en 1993 (cette fois-ci, avec une assislante de recherche), pour y effectuer une étude de type plus qualitatif d une durée de deux mois. au cours de laquélle on a rencontré 71 travailleurs de tous niveaux qui étaient déjà à l'emploi de l'entreprise en 198.3. soit $41.8 \%$ des anciens travailleurs de 198.3 qui restent. L'étude adopte une approche méthodologique mixte. combinant: 1) des enregistrements d'entrevues individuelles, semi-dirigées; 2) des questionnaires mixtes, soumis oralement et enregistrés, qui reprennent les thèmes des entrevues, et qui sont complétés par des enregistrements libres en situation naturelle de travail: 3) quelques questionnaires écrits, auto-administrés, de type fermé, reprenant les thèmes des entrevues; et enfin, 4) de lobservation sur place. La durée des enregistrements varie entre $3 / 4$ d heure pour les questionnaires et 1 heure $1 / 2$ pour les entrevues, pour un total d'environ 1 I.5 heures.

\section{Caractéristiques de l'entreprise étudiée}

Il sagit d'une entreprise essentiellement francophone, tant par la langue première des répondants. que par le fait que la langue habituelle de travail y est le français ${ }^{10}$. II s'agit donc là d'une situation idéale pour examiner la pénétration et l'influence de l'anglais dans un contexte optimal, en principe du moins, pour l'utilisation et la diffusion du français. De plus, au moment de la première cueillette de données en 1983. I'entreprise venait de mettre sur pied une campagne de diffusion et d'implantation de terminologie de langue française, êt avait déjà sensibilisé ses employés à la question, en distribuant, entre autres. un lexique des termes techniques propres au domaine. Certains changements importants ont cependant eu lieu entre 198.3 et 1990-93. Tout d'abord, la campagne de francisation terminologique ne s'est pas poursuivie au-delà de la deuxième année. bien que la haute 
direction ail toujours encouragé l'utilisation du français prour les termes lechniques. Ensuite. lentreprise a subi plusieurs transformations organisationnelles importantes dans le cadre de fusions avec d'autres entreprises québécoises du même secteur. Malgré tout. les données de 1990 et de 1993 comprennent un nombre suftisamment important d'anciens travailleurs qui ćlatient déja a l emploi de l'entreprise en 198.3 pour permettre des comparaisons systématiques. De plus. les renseignements obtenus en 1993. Lors de l'étude de type qualitatif. permettent une analyse pouscée de lat situation. It faut souligner cependant que les analyses statistiques des donnees de 1990 ne sont pas encore completees. Quant aux donnees de 1993. elles commencent a peinc à etre codifiée. Il va denc sam dire que les résultats présentés ici sont préliminatires".

\section{La méthodologie}

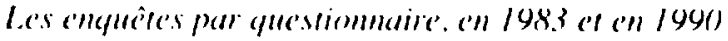

1.e questionnaire distribue en 1983 el en 1990) fournit des renseignements sur:

$1)$ des facteurs sociodémographiques: age, sexe, langue première et langue d'usage. niveau de scolarité, langue des éludes générales. langue de la terminologie en milieu d'apprentissage, atc:

2) des facteurs occupationnels el organisationnels ou communicationnels: poste. canal de communication principal au travail (l'oral ou l'écrit). réseaux communicationnels (communications hiérarchiques, inter et intra-entreprise. au Québec et hors du Québec, etc.):

3) la perception générale des répondants par rapport à leur comportement terminologique dans plusicurs types de communications orales et écrites et dans différentes situations. hiérarchiques et organisationnelles:

4) certaines opinions et comportements révélateurs d'attitudes des répondants par rapport à la capacité du français et de l'anglais à exprimer les réalités lechniques: par rapport au processus de frantisation en général, sa faisabilité, ses avantages et ses désavantages. tant sur le plan personnel qu organisattionnel: le niveau de conscience du fait qu une campagne de francisation soil en cours (ou ait cu tieu. e'n 1990) et les opinions par rapport à son bien-fondé el alux résultats escomptés (ou obtenus. en 1990): l'engagement personnel dans le processus de francisation de l'entreprise et du Québec en général: le niveau de conscience du fiat qu un changement linguistique soit en cours et la perception qu ${ }^{\circ}$ on se fait de ce changement, tant dans son propre comportement que dans celui de son entourage, etc. Le questionnaire de 1990 comprend. de plus, des opinions el perceptions par rapport all changement linguistique et sociolinguistique des dernières années. de même qu une évaluation de ce changement.

5) Enfin. les lecuteurs sont confrontés à un choix entre une série de 10 termes techniques de langue française et leurs équivalents de langue anglaise, tant pour l'oral que pour l'écrit. ce choix élant considéré comme un indice du comportement terminologique «réel».

Cette approche méthodologique a permis de recueillir un grand nombre de données standardisées el comparables - dans le temps - el ce. auprès d'un nombre important de répondants. De plus, la confrontation de chacun des blocs de varriables retenues (les variables sociodémographiques. occupationnelles et organisationnelles ou communicationnelles, les perceptions générales sur la situation et le comportement terminologiques, à loral et à lécrit. les opinions révélatrices d'attitudes. et le choix des langues pour les termes testés, a l'oral et a l'écrit). minimise le biais inhérent à toute enquête d'opinions. el assure une meilleure tiabilité des résultats. 
En ce qui concerne les termes techniques, il va de soi que les questionnaires ne permettent pas d'avoir accès au comportement terminologique réel. mais uniquement au comportement déclaré. Cependant, même si l'idéal aurait été de procéder à des enregistrements en situation rélle, une telle approche $n$ 'aurait pas permis de récolter des données terminologiques comparables pour tous les sujets, et ce, tant pour l'oral que pour l'écrit. De même, il aurait été certainement quasi impossible de procéder à une cueillette comparable de données enregistrées quelques années plus tard.

Sur ce point, signalons que, sans doute, ni le choix entre des termes français et leurs équivalents anglais présentés de façon explicite. ni la perception générale qu'ont les répondants de leur comportement terminologique dans différentes situations de travail ne reflètent exactement le comportement linguistique réel d'un individu en situation naturelle. En fait. il est probable que les perceptions qu'ont nos répondants de leur comportement linguistique reflètent plutôt un consensus social et culturel par rapport à un modèle linguistique et sociolinguistique (voir entre autres, Bourhis 1983, 1984: Edwards 1985: Labov 1972h; Lambert 1967: Milroy 1980; Shuy et Fasold 197.3). II reste qu'on peut raisonnablement présumer que le choix concret de termes techniques spécifiques, même en contexte artificiel, reflète davantage le comportement linguistique réel, du moins pour ces termes, que les perceptions générales que se font les locuteurs de leur comportement linguistique et terminologique global en situation de travail. On reviendra d'ailleurs sur ce point.

Pour ce qui est des 10 termes testés, ils ont été choisis par l'entreprise qui voulait voir jusqu à quel point on pouvait les franciser dans le cadre de leur campagne de francisation. Il sagit de termes d'emploi généralisé. bien implantés dans le milieu. mais dont certains posaient problème (en 1983 et encore en 199(1-9.3) par rapport aux efforts de francisation. Ils se situent donc à l'extrémité du continuum terminologique où on trouve les termes réfractaires à la francisation, et dans ce sens, ils servent de barème.

\section{L'enquête de type qualitatif de 199.3}

D’ailleurs. les lacunes inhérentes à l'approche méthodologique d'enquêtes par questionnaires sont minimisées par l'étude de type qualitatif, effectuée en 1993. Cêlte nouvelle étude a permis de collecter un grand nombre de termes techniques pour chacun des répondants ${ }^{12}$. Les termes techniques sont classés, entre autres, selon différents niveaux de technicité. allant des termes utilisés pour l'administration générale aux termes propres au transport routier ${ }^{13}$. Bien entendu, nous testons de nouveau les 10 termes techniques testés en 198.3 et en 1990 de façon à pouvoir comparer les résultats.

Les autres renseignements recueillis sont comparables à ceux obtenus par les questionnaires d'opinions (de 198.3 et 1990), mais contiennent en plus un grand nombre de renseignements supplémentaires qui permettront une analyse poussée. De plus, ces renseignements sont complétés par les commentaires et explications métalinguistiques et sociolinguistiques obtenus au cours d'entrevues ou par l'observation sur place. L'ensemble des données de 1993 seront. dans la mesure du possible, codifiées pour lin danalyses statistiques dans le but de comparer systématiquement les variables des trois corpus 1 (198.3. 1990 et 1993). et de véritier. en les confrontant à la situation naturelle dans l'entreprise. certaines hypothèses retenues à la suite de l'analyse des données de 198.3 et de 1990 .

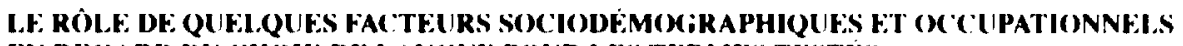 EN RF.(;ARI) DU ( HOIX DE L.ANGIH: POUR LES TERMES TESTES}

Examinons maintenant quelques facteurs scociodémographiques et occupationnels qui ont joué un róle important en 1983. en regard du choix de langue pour les 10 termes 
techniques testés. En confrontant la situation terminologique el sociolinguistique de 198.3 à celle de 1990. on tentera de dégager l'importance de ces mêmes facteurs dans le processus du changement terminologique et sociolinguistique. $S$ 'il s'avérait que certains d'entre eux semblent jouer un rôle systématique en 1990 comme en 1983, on pourrait vraisemblablement en déluire qu ils constituent des éléments dont on devrait tenir compte dans une éventuelle politique d'implantation terminologique. Rappelons que les analyses statistiques des données de 1990 en sont encore au stade préliminaire et que les résultats présentés ici constltuent une première analyse.

Après avoir présenté une vue d'ensemble de la situation terminologique déclarée, dans l'entreprise, en ce qui a trait au choix du français ou de l'anglais pour les 10 termes techniques testés, en siluations communicationnelles, à l'oral, on examinera cés mèmes données en fonction de certaines catégories de travailleurs pour voir si chacun de ces groupes se componte de la même façon. Plus précisément. on essaiera de voir si le niveau de scolarité. le sexe et le poste occupé ont une incidence sur le comportement terminologique déclaré. Par la suite. on tentera de voir si certaines opinions révélatrices dattitudes. par rapport au processus de francisation, permettent de comprendre la situation terminologique et sociolinguistique de l'entreprise.

\section{La situation de 1983}

Mais, auparavant. examinons la situation de départ dans l'entreprise, en 198.3. Quelle est la situation terminologique, telle que perçue par les travailleurs? Et surtout. est-il possible d'identitier des facteurs extralinguistiques qui pèsent sur le choix déclaré des langues, en l'occurrence du français, pour les termes testés"?

Sur ces questions. l'analyse des données de 1983 tend à démontrer que la plupart des variables sociodémographiques, occupationnelles et communicationnelles lestées. comme lage, le sexe, le niveau de scolarite, le poste et le canal de communication (utilisation de l'oral ou de l'écrit au travail) jouent un rôle en regard du choix déclaré de langue pour les termes techniques testés, quand on les prend en comple individuellement (test du khi carré). Cependant. le rôle qu'elles jouent n'est pas d'égale importance, quand on regarde l'ensemble de cés variables en fonction du choix de langue déclaré. En fait. il s'est avéré que, mis à part le niveau de scolarité. le poste (et dans une certaine mesure le canal de communication), qui conservent toujours une valeur explicative importante quelles que soient les analyses effectuées, les autres variables sociodémographiques ont un rôle moins important qu'on l'aurait cru, quand on examine simultanément, dans le cadre d'analyses de régressions. les différentes catégories de variables testées: c'est-i-dire. les variables sociodémographiques, occupationnelles/communicationnelles et certaines perceptions et opinions révélatrices d'attitudes (Daoust 1988 et 1989h) ${ }^{14}$. En fait, ce sont justement certaines perceptions et opinions révélatrices d attitudes, de même que certains types particuliers de comportements révélateurs d'attitudes, couplés à certaines variables comme le poste et le niveau de scolarité, qui rendent mieux compte du comportement terminologique déclaré.

Par ailleurs, les analyses statistiques effectuées ont démontré qu il existe à la fois un lien et un écart systématiques entre, d'une part. certaines perceptions générales de la situation terminologique ét certaines opinions révélatrices d’attitudes par rapport au français el a la francisation et. d'atutre part. le choix explicite des termes testés. Cela revient a dire que tous les répondants, quelles que soient les catégories de classement utilisces. déclarent des perceptions, opinions ou comportements révélateurs d'attitudes plus favorables au français que ne le laisserait croire leur choix du français pour les lermes techniques testés. Celle double relation systemattique varie en fonction de variables sociodémographiques et organisationnelles/occupationnelles comme le sexe. le poste. l'âge et 
la scolarité. Concrètement, ceci revient à dire que. par exemple, les femmes, les travailleurs les plus instruits, et les titulaires de postes de gestionnaires et de cols blancs ont des perceptions et des opinions moins aexagérées" de leur utilisation déclarée du français pour les termes techniques testes que les hommes, les travailleurs moins instruits et les cols bleus. Mais on reviendra sur cette question.

\section{Le choix de langue pour les termes techniques testés, à l'oral}

Examinons maintenant la situation terminologique telle que perçue par les répondants. Le tableau suivant (1) présente, sous forme de pourcentages contrastés, le choix déclaré des langues pour les 10 termes testés, pour l'ensemble des travailleurs de 198.3 el pour le groupe de travailleurs de 1990 qui étaient déjà à l'emploi de l'entreprise en l98.3 (le groupe "A"). La colonne de droite présente les mêmes données sous forme de scores. calculés à partir des pourcentages, et pondérés en faveur du français, de façon à former une échelle continue de 0 à 100 . Ces scores, que je désignerai sous le nom d' andices de francisation". permetlent davoir une vue densemble de la situation en fonction du français.

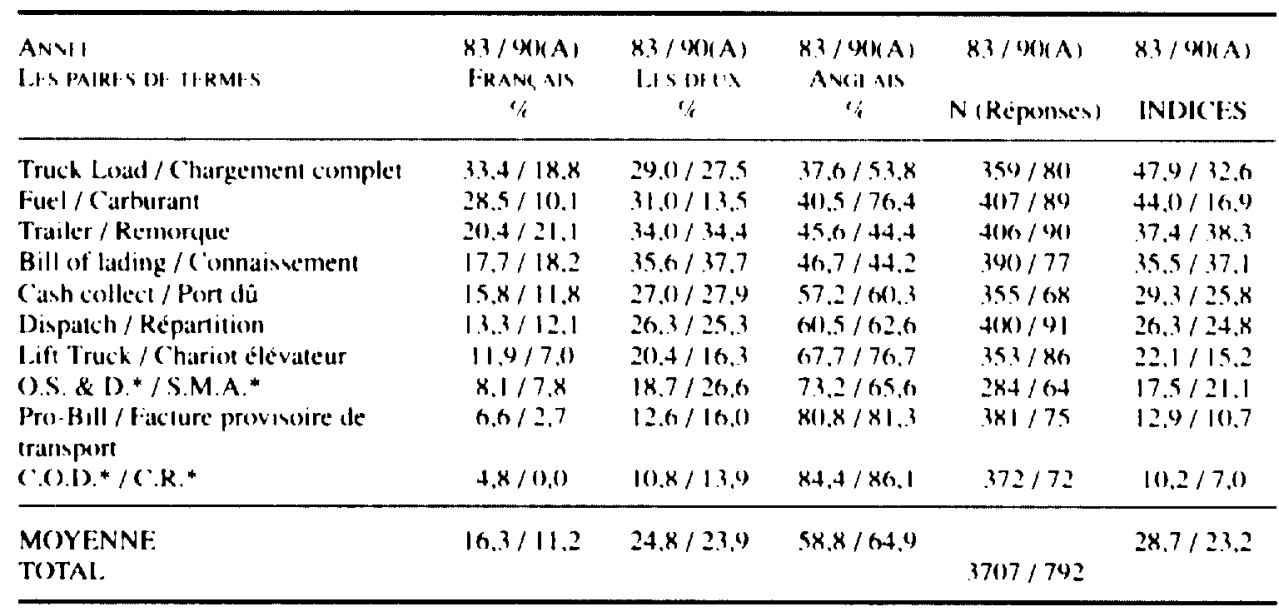

Tableau 1. L'entreprise de transport (198.3 et I99()A): la comparaison du cholx de la langue pour les 10 lermes techniques. Fréquences generales deutisation dans les communicalions orales. Comparaison entre cous les

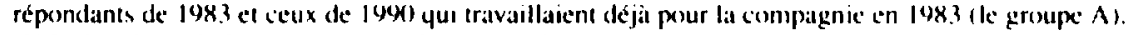

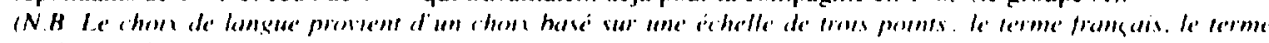

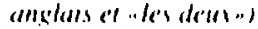

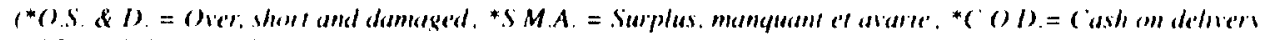

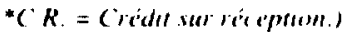

Indices de francisation: $1 / 2(2 \times \%$ Terme français + $/ /$ les deux $)$

1.indice moyen a elét obtenu en appliquant la formule precedente sur les pourcentages moyens

Seuls sont anclus dans le calcul ceux yuu se sont prononces sur cette yuestion

Comme on peut le constater par les moyennes générales autant que par l’indice moyen de francisation, l'ensemble des répondants déclare faire un usage assez important de l'anglais, tant en 1983 qu'en 1990. À première vue, on remarque même une augmentation de l'utilisation déclarée de l'anglais en 1990 . Ceci ressort de la moyenne générale pour le choix de l'anglais, qui est de $58.8 \%$ en 1983 . contre $64.9 \%$ pour le groupe des anciens travailleurs (groupe A) en 1990 . De même. la moyenne des termes techniques 
que les répondants déclarent utiliser en français uniquement est passée de $16.3 \%$ pour les employés de 1983 a $11.2 \%$ pour ceux du groupe A en 1990. Cetle baisse déclarée de Putilisation du français semble favoriser soit une plus grande utilisation de langlais, soit l'alternance entre le français el l'anglais, la moyenne générale pour celte dernière catégorie ctant cependiant restéc relativement stable $124.8 \%$ en 198.3 contre $23.9 \%$ pour le groupe $A$ de 1990). Si l'on compare maintenant les indices de francisation, on remarque que l'indice moyen a chute de 5.5 points, pour atteindre $23.2 \mathrm{en} 1990$ contre $28.7 \mathrm{en} 198.3$.

Cependant, un examen plus attentif révele qu' il y at une grande variation au niveau des termes eux-mémes, en 1983 comme en 1990, cee qui rend comple. en partic, de la diminution du choix déclaré du français pour ces termes. Il est clair qu une bonne partic des termes de langue française que l'entreprise a voulu promouvoir en $1983 \mathrm{n}$ a pass été acceptée par les employes. Ce sont ces termes qui rendent compte de la progression de l'anglais. ("est le cils, entre atutres, de "C.R." veruss "C.O.D.", "chargement complet/ charge entiere" versus atruck load" et "arburant" versus afuel». Malgré tout, sauf pour "remorque" et "connaissement". ke choix du français comme seul choix a diminué pour tous les lermes lestes. ("ent ce qu'on constate par les moyennes d"utilisation exclusive du français. Dans certiains cais, cependant. celle diminution se traduit plutôt par une augmentation de l'alternance entre le français el l'anglais, qu'on pourrait peut-être interpréter. sous toute réserve, comme un phénomène de bilinguisation.

Il serait tentant, à ce stade, de conclure à un échec relatif de la campagne de francisation terminologique de 1983. Pourtant. un examen plus approfondi des données de 1990. de même que lès renseignements provenant de l'élude effectuée sur le terrain. en 1993. incitent à la prudence. Ainsi. il ressort clairement de ces dernières données, que. tel que mentionné, les 10 termes testés en 1983 el en 1990 sont effectivement des termes fréquemment utilisés ou du moins connus par la presque totalité des employés de lentreprise. el que, surtout. cee sont bel et bien des termes-problemes pour la francisation. el ce. encore en 1993. Ce sont. aux dires d'une majorité de travailleurs interrogés en 1993. des termes anglicisés de longue date et réf ractaires à la francisation. et qui. selon certains, ne seront jamais francisés. Une chose semble claire. ils se situent effectivement au point le plus anglicise du continuum terminologique. Its ne sont pas représentatils de l'ensemble des termes utilisés, mais bien plutôt du noyau dur de termes réfractaires à la francisation. Par contre, ils somt importants de par leur fréquence d'utilisation. Avant de conclure à un recul du français, il faudrait prendre en compte l'ensemble des termes recueillis en 199.3 de façon relativement spontanće parmi lesquels on dénombre de nombreux termes en français. tout en pondérant ces données selon la fréquence d utilisation ${ }^{15}$.

Par ailleurs, il semble aussi qu'il faille se méfier de certaines interprétations. Par exemple. la paire de termes testés: "fuel/carburant" n'est pas en réalité complètement synonymique. Ainsi, afuel» semble être un terme spécialisé qui désigne le carburant pour diesels. Dans celle acception. il $n$ est pas un anglicisme pur et simple en variation avec le mot «carburant". qui. lui. semble être à la fois un générique et un synonyme du mot "essence". Par ailleurs, il faut aussi tenir compte du fait que certains de ces termes identifiés comme des termes anglais ne sont pas nécessairement perçus comme tels. Le cas de "C.O.D." constitue un exemple frappant de celte situation. En effet. ce terme semble à ce point integre au français que certains travailleurs rencontrés en 1993 ne connaissent pas le terme de depart "cash om delivery" et ne semblent pas le considérer comme un terme anglais. Et mêtme, parmi ceux qui savent que le terme est anglais à l'origine, ils semblent nombreux à l'accepter comme un terme du français québécois, même si, parfois ils déplorent le fait que ce soit un anglicisme. Il faut donc être très prudent lorsqu 'il s'agit d'interpréter ce type de données. Mais laissons là cette question de l'évaluation de la situation terminologique, yui ne constitue pas, à proprement parler. notre propos principal, et essayons 
de voir si le schéma de compontement terminologique présenté ci-dessus est le même pour routes les catégories de travailleurs. En fait, telle que mentionnée. l'analyse des données de 198.3 a révélé que le comportement terminologique déclaré variait en fonction. entre autres, du niveau de scolarité, du sexe et du poste occupélt. Mais, est-ce que ces mêmes variables jouent toujours le même rôle en 199() ?

\section{La relation entre le comportement terminologique déclaré, à l'oral,}

et le niveau de scolarité, le sexe et le poste

La figure suivante présente les données sur le choix déclaré de langue pour les termes techniques testés. dans les communications orales. en fonction du niveau de scolarité des répondants. Les résultats sont présentés sous forme d indices ${ }^{17}$.

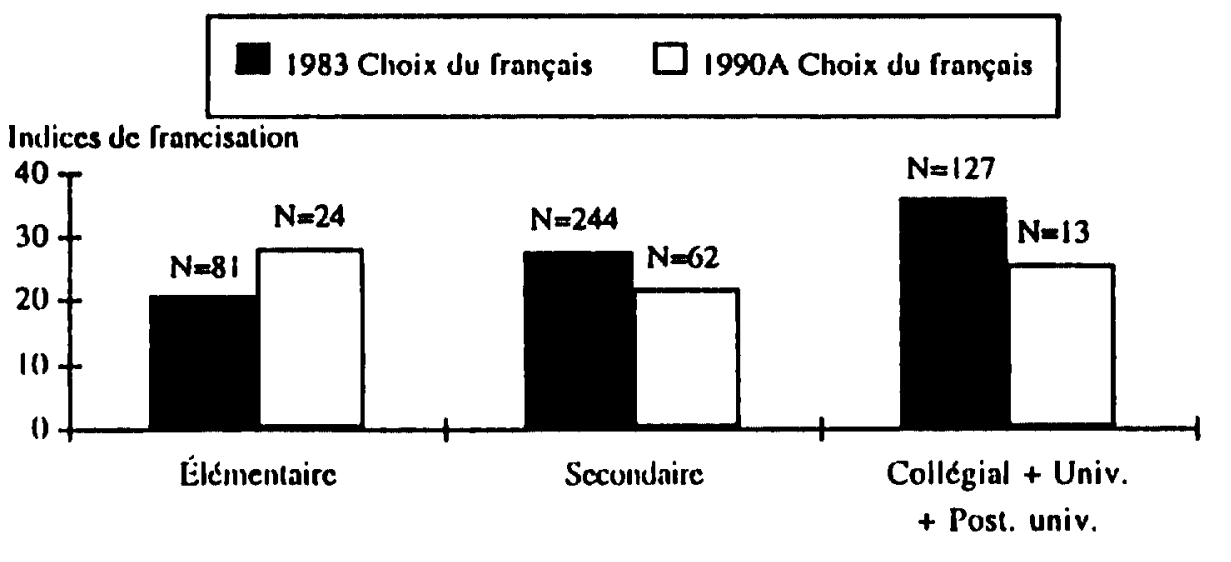

Figure 1. Lentreprise de transport ( 198,3 et $I 9(x)$ A): le choix declare de langue pour les 10 termes kechniques testes. dans le contexte des communcations orales, en fonction du niveate de scolarite.

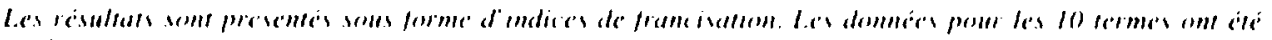
anghemingies.

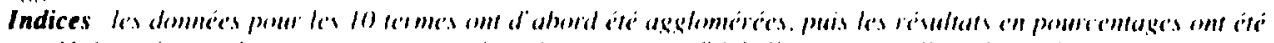

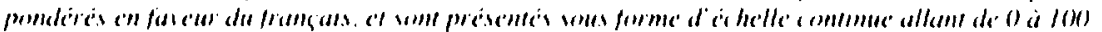

Indice de francisation $1 / 2(2 \times 1 /$ terme trançais $+/ / /$ les deux $)$

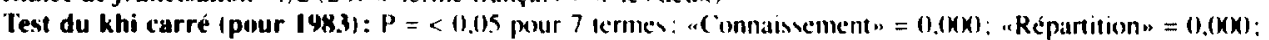

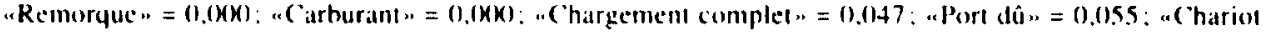

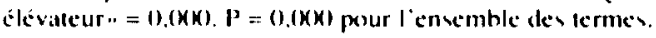

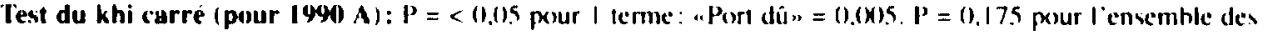
tcruluss.

( $N=$ le tonal des répondant dans cen categories, qui ont participe a l'enquête)

Seus sont inclus dians te calcul ceux qui ve some pronences sur celle question.

Il ressort clairement que. en 198.3. plus les travailleurs sont instruits. plus ils déclarent un comportement terminologique favorable au français. Cette relation entre le niveau de scolarité et le choix des langues pour les termes testés s'est avérée statistiquement significative. comme l’indiquent les résultats du test du khi carré inscrits au bas de la figure I pour le corpus de 198.3 ( $P=<0,05$ ). Si l'on regarde maintenant la situation de 1990), on se rend compte que. contrairement à ce qui se passait en 198.3. les travailleurs se répartissent en deux groupes: les travailleurs les moins instruits (sur la gauche de la figure) et 
les autres. Celle fois-ci. ce sont les travailleurs les moins instruits qui déclarent le plus important taux d'utilisation du français pour les termes testés. Voilà qui est assez surprenant. Par contre. pour ce qui est des autres catégories de travailleurs de 1990, on peut dire que. mis à part le fait que le choix du français est moins important qu'en 1983. le schéma indiquant le choix du français suit sensiblement la même courbe qu'en 1983. Les travailleurs les plus instruits. cest-ì-dire ceux qui ont fait des éludes de niveau collégial ou universitaire. déclarent un comportement terminologique plus favorable au français que leurs collègues qui n'ont pas dépassé le niveau secondaire. $\dot{A}$ ce stade-ci des analyses, il est difficile de fournir une explication qui rendrait compte du fait que les travailleurs les moins instruits ont l'indice de francisation le plus élevé. Les analyses statistiques effectuées sur ces données de 1990 A (test de khi carré) ne permettent pas de conclure que le niveau de scolarité a un impact sur le choix de langue pour les termes testés. Mais. comme le niveau de scolarité semble jouer un rôle important à d'autres niveaux. on retient que la situation terminologique semble avoir évolué entre 198.3 et 1990) et que. tout comple fait. les travailleurs les moins instruits sont ceux qui semblent avoir fait le plus de "progrès" par rappon à la francisation. en ce qui concerne les termes testés, alors que les autres accusent un léger recul sur ce point. Cependant, il faudra effectuer d'autres tests statistiques avant de se prononcer sur l"importance de ce facteur pour 1990 $\mathrm{A}^{1 \mathrm{x}}$.

Cependant, quelle que soit la valeur statistique de la relation entre le niveau de scolarité et le comportement terminologique déclaré. les résultats montrés à la figure 1 indiquent clairement que toutes les catégories de travailleurs $n$ 'adoptent pas un comportement terminologique identique. Si l'on veut vraiment comprendre la dynamique sociolinguistique de cette entreprise, on ne peut done pas se fier uniquement aux données globales présentées dans lé tableau 1 , où toutes les catégories de travailleurs sont confondues. Il faut plutôt essayer de comprendre le comportement de différentes catégories de travailleurs.

Poursuivons donc dans ce sens et regardons maintenant si le sexe joue un rôle dans le choix déclaré du français pour les termes techniques testes. en contexte communicationnel oral.

Comme on peut le voir dans la ligure 2, les femmes, tant en 198.3 quen 1990 (groupe A), ont un indice de francisation beaucoup plus élevé que les hommes. Le comportement terminologique déclaré semble donc être en corrélation très forte avec le sexe. ce que corroborent les tests statistiques effectués (voir au has de la figure. les résultats du test de khi carré). Il ressort clairement que les femmes choisissent davantage le français pour les termes techniques testés que les hommes, ce qui permet de conclure que les femmes favorisemt davantage la forme normative. (ees résultats. obtenus en milieu de travail. corroborent ceux d'autres recherches de type sociolinguistique effectuees dans des contextes non reliés au travail. Ainsi, des chercheurs comme Milroy (1980) et Labos (1972b) ont trouvé que les fermmes avaient tendance à utiliser divantage de formes normatives, ou perçues comme prestigieuses sur le plan social, que les hommes. Dans le contexte de l'entreprise québécoise, ou la Charte de la langue française prescrit l'utilisation d'une terminologie de langue française, on est justifie de croire que les termes techniques de langue française somt perçus comme valorisés socialement, ou tout au moins. comme étant conformes a la norme. Si tel est le cas, il est possible que les travailleuses de l"entreprise étudiée soient a l'avant-garde d'un mouvement qui favorise une plus grande utilisation du français pour les termes techniques. Bien sûr, less résultats comparatifs de 198.3 et de 1990 présentés dans la ligure 2 ne permettent pas de conclure qu il y att eu un changement à une plus grande utilisation du français: cependant, ils ne permettent pas non plus de conclure à un recul important du français en 1990. Ce qui semble certain. cest que, toute proportion gardée, la tendance vers une plus grande utilisation du français 


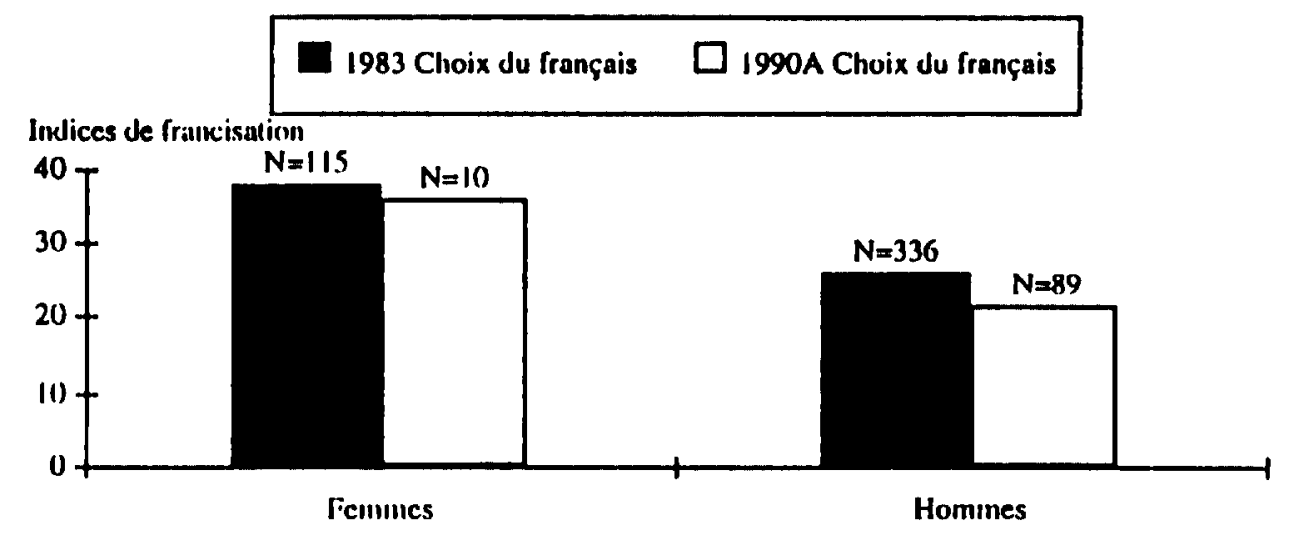

Figure 2. Lientreprise de transport $(1983$ e 1690 ) Al: le choix declare de langue pour les 10 termen techniques

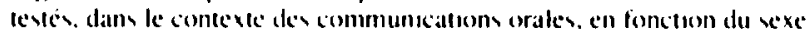

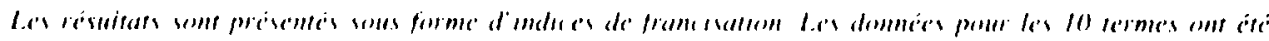
asphemirion.

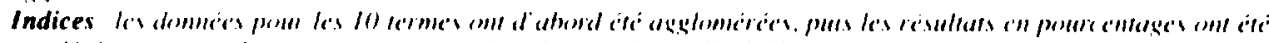

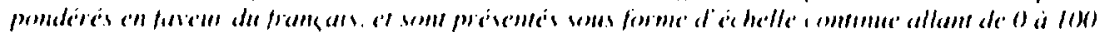

Indice de francisation $1 / 212 \times$ " terme frantian + " les deux

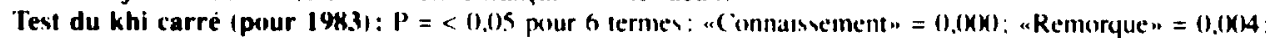

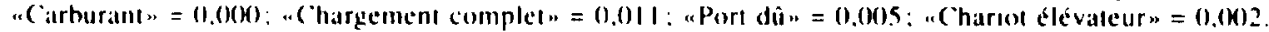
$P=0,($ Kno pour liensemble des lermes

Test du khi carre (pour 1990 Al: $\mathrm{P}=<0.05$ pour 3 termes: "S.M.A. $=0.0013$ : "Carhurant" = 0.004: "Chargement complet" $=0,010 . P=0 .(K)$ ) pour l envemble des lermes.

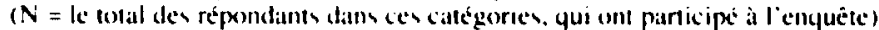

Seuls sent inclus dans le calcul ceux qui se som prononcés sur cette question.

par les femmes se maintient en 1990. Les femmes sont donc, en 1990 comme en 1983. à l'avant-garde de la francisation terminologique de leur entreprise.

Cependant, encore une fois, il faut interpréter ces résultats avec prudence. Étant donné que le contexte social de l'entreprise est différent de celui de la société en général. et que les femmes en milieu de travail ne jouent pas le même rôle familial et social qu elles jouent ailleurs, il est possible que ce comportement qui semble lié au sexe, le soit en fait. particllement du moins, aux fonctions qu'occupent les femmes dans l'entreprise. En effet. dans l'entreprise éludiée. il y a une étroite corrélation entre le sexe et les postes occupés. Ainsi, en 1983. toutes les secrétaires $(N=28)$ étaient des femmes ${ }^{10}$. Quant à leurs fonctions, il est bien connu que les employées de secrétariat sont souvent responsables de la qualité du français dans leur entreprise. et même. partiellement du moins. de la francisation de l'entreprise, surtout pour ce qui est de l'écrit. Dans ce sens, la direction de l'entreprise exerce une pression sur ces employées, qui les rend peut-être davantage conscientes de l'importance d'utiliser le français. Les secrétaires interviewées en 1993 ont confirmé ceci. Il est donc plausible de croire que c'est cette pression liée à leur fonction. autant ou peut-être plus que le sexe, qui explique le fait que les travailleuses semblent favoriser le français plus que leurs collegues masculins. Mais on reviendra sur ce point.

Regardons maintenant les données sur le choix déclaré des langues pour les 10 termes techniques en fonction de l'occupation. Tous les travailleurs ont été regroupés en six catégories occupationnelles ${ }^{21}$. 


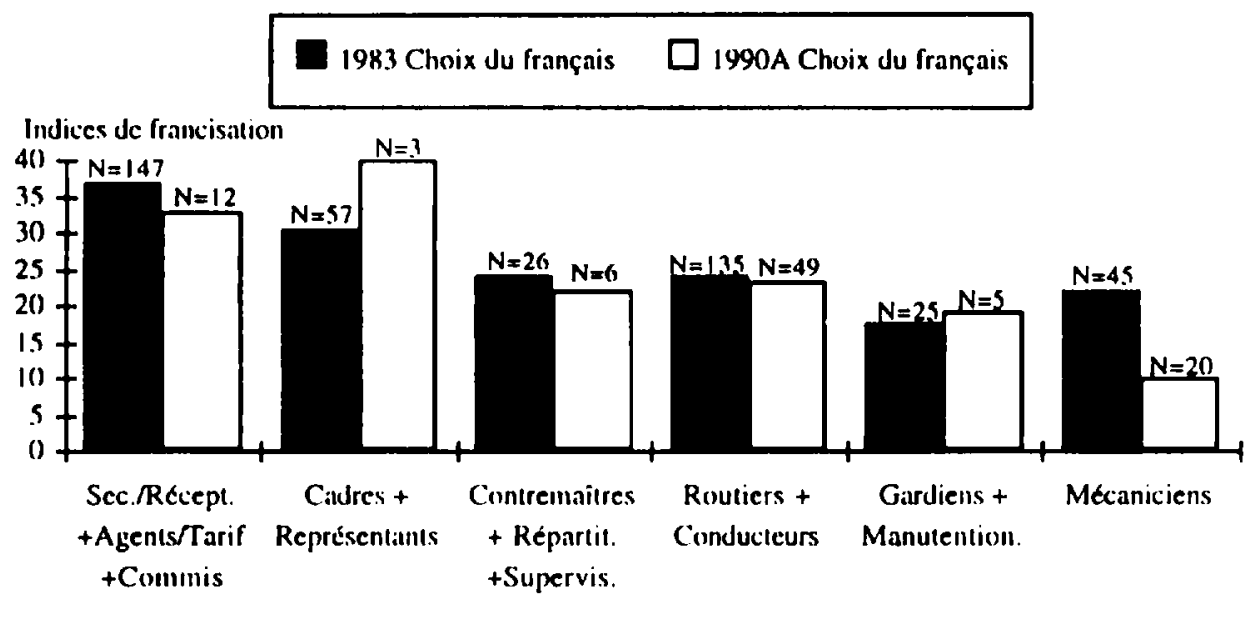

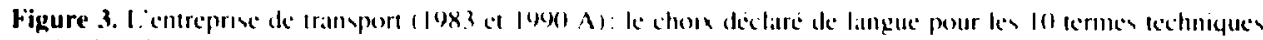

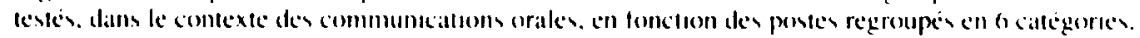

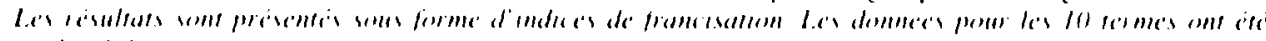
agelemeirices

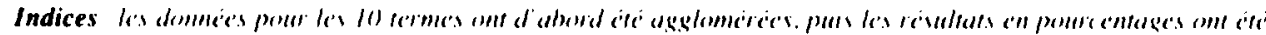

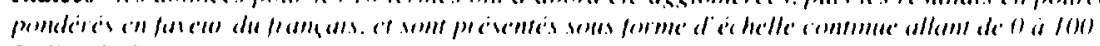

Indice de francisation $1 / 212 \times$ "s terme transals + "Fendeun

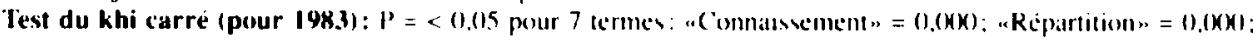

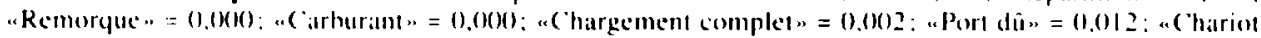

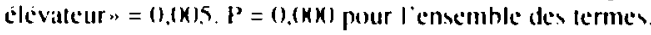

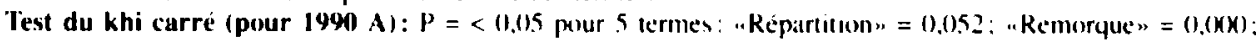

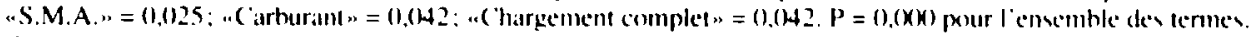

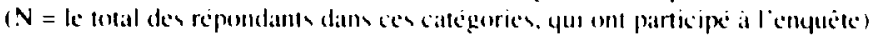

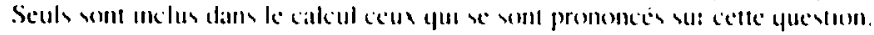

Il ressort clairement de la figure 3 que. en 1990 comme en 1983. les travailleurs forment deux groupes distincts. Ie premier groupe, à gauche de la figure, formé des cadres et gestionnaires. de même que des employés de secrétariat et de bureaa. déclare un emploi relativement important du français pour les termes testés. Le deuxième groupe. à droite de la figure. est caractérisé, pour sa part, par un emploi déclaré du français bealucoup moins important. Ce groupe comprend les travailleurs exerçant des fonctions de cols bleus ou de supervision de second niveau. Si l'on compare maintenant la situation de 1983 avec celle de 1990 (pour le groupe A), il est intéressant de noter que. même si lion remarque une tendance à une légère diminution de l'utilisation déclarée du français pour les 10 termes techniques, cette tendance est loin d'être importante. sauf pour le groupe des mécaniciens. dont l’indice de francisation a chuté de plus de lo points, pour passer de 22,0 en 1983 a 10,2 en 1990. Cela est contrebalancé par le fait que les cadres. gestionnaires et représentants déclarent. de leur côté. une utilisation accrue du français en ce qui a trait aux termes testés. En effet. leur indice de francisation a augmenté de 10 points pour passer de 30,4 en 198.3 à 40,0 en 1990 .

Quant aux secrétaires el autres employés de bureau. la figgure 3 révèle qu ils avaient l'indice le plus élevé en 1983: 36.8. Fin 1990, ils se situent au deuxième rang, avec un indice de 32.9. Encore une fois la question se pose, à savoir si ces résultats sont attri- 
buables au poste oxcupé ou au sexe. On a vu plus haut que les femmes déclaraient un usage plus important du français pour les termes testés que les hommes. et que. par ailleurs. les secrétaires étaient toutes des femmes $(N=28)$. Mais il y a d'autres postes qui sont étroitement corrélés au sexe. Par exemple. les postes de cadres, de gestionnaires, de chauffeurs de camion, de mécaniciens, etc., sont l'apanage quasi exclusif des hommes. Par contre, sauf pour le poste de secrélaire, les deux sexes sont représentés dans les postes d'employés de bureau, même si les femmes sont plus nombreuses à occuper ces postes que les hommes. Ainsi, en $1983,65 \%$ des commis $(N$ total $=89$ ) et $54 \%$ des agents d'administration ( $N$ total $=26$ ) étaient des femmes, ke reste, des hommes. En 199), le nombre total de répondants est trop petit pour risquer une analyse de ce genre. Ainsi, on ne compte que 9 commis et agents d'administration et, sur ce nombre, un seul employé est une femme. Quoi qu'il en soit, en 198.3 comme en 1990, il est clair que les travailleurs occupant des postes de bureau sont ceux qui déclarent un des taux les plus élevés de français. Et. étant donné qu un nombre relativement important d'hommes détiennent ces postes. il devient assez délicat d'attribuer ce comportement au seul facteur sexe. Il apparaît donc justitié d'y voir une combinaison des deux facteurs.

Mais. quelle que soit la corrélation entre les variables sexe et poste. il semble clair que le statut du français, tout au moins en ce qui concerne le comportement déclaré des divers groupes de travailleurs par rapport aux 10 termes, est relativement semblable dans les deux corpus de 1983 et de 1990. En 1983. Les analyses statistiques ont démontré que le choix déclaré de langue pour les termes testés était fortement corrélé au poste, et que de ce fait. le poste occupé constituatit un indice fiable du choix du français - ou de l'anglais - pour les terme's testés. Il en est de même pour le corpus de 1990 (groupe A). ainsi qu en témoignent les résultats des tests du khi carré inscrits au bas de la figure 3.

Par ailleurs, les résultats présentés à la figure 3 semblent indiquer que la siluation terminologique. telle que perçue par les différents groupes de travailleurs est encore, toute proportion gardée, pro-française en 1990, surtout si l'on prend en compte les changements organisationnels importants qui ont eu lieu entre 198.3 et 1990. et le fait que la campagne de francisation entreprise en 198.3 ne se soit pas poursuivic au-delà de 1984. De plus, si. comme semblent le croire bon nombre de travailleurs interrogés en 199., les 10 termes testés sont des termes réfractaires à la francisation. on peut croire que le français a au moins gardé le statut qu il avait en 1983. Peut-être même la situation s'est-elle améliorée. En fait. les analyses préliminaires du nouveau corpus de 1993, qui comprend un nombre très important de termes techniques autres que les 10) termes testés en 1983 et 1990. permettent d'envisager cette hypothèse. Évidemment, on ne pourra se prononcer sur ce point qu après avoir analysé à fond ces nouvelles données de 1993.

En altendant, que peut-on conclure des résultats comparatifis de 1983 et de 1990 ? Eh bien. si l'on regarde au-delà des données générales présentées dans le tablèau I qui résume la siluation de l'entreprise, toutes catégories de travailleurs confondues, il semble clair qu'on ne peut comprendre la situation terminologique et sociolinguistique que si l'on examine le comportement des différents groupes de travailleurs. lees données des figures 1,2 et 3 montrent qu il existe des groupes de travailleurs qu'on pourrait qualifier de pro-français. Qui sont-ils? Ce somt ceux qui occupent des postes de gestionnaires et d'employés de bureau. Une partie de ces mêmes travailleurs se trouvent être des fémmes. de sorte qu'on peut dire que le poste et le sexe semblent être deux facteurs qui jouent sur le choix déclaré des langues pour les termes techniques lestés. Le rôle de ces deux facteurs est constant en 1983 et en 1990. Quant au niveau de scolarité, son rôle reste à déterminer par rapport à la situation de 1990): il s'est révélé cependant très important en 1983. Il y a bien sûr d'autres facteurs, comme l'âge et le canal de communication. qui semblent liés au comportement terminologique déclaré. ainsi qu on l'a dit plus haut. 
LINTERRELATION ENTRE I.E CHOIX DE LANG'UE DÉCLARE POUR I.ES TERMES DANS I.E CONTEXTE DES COMMUNICATIONS ORALES FT QUELQULS OPINIONS REVEI.ATRICES I) ATTITUDES PAR RAPPORT À I.A FRANCISATION FEN FONCTION DU POSTE FT DI SEXE:

Par ailleurs, comme on l'a déjà mentionné, les analysés effectuées sur les données de 198.3 ont révélé que le comportement terminologique déclaré dés différents groupés de travailleurs était aussi en corrélation statistiquement significative avec certaines opinions ou comportements déclarés, révélateurs d'attitudes. Par exemple, en 1983, les travailleurs les plus instruits non seulement déclarent un taux plus élevé d'utilisattion déclarée de termes français pour les termes testés que leurs collegues moins instruits, mais semblent aussi entretenir des opinions plus favorables pour le français et le processus de francisation. L 'inverse est vrai, de sorte qu on peut dire que. tout comme le choix déclaré des langues pour les termes testés est en corrélation avec le niveatu de scolarité, il en est de même pour certaines catégories d'opinions ou de comportements révélateurs d'attitudes. En 1990, il semble que l'on puisse aussi établir un lien statistiquement significatif entre la scolarité et certaines opinions révélatrices d'attitudes. Cependant. en 1990). contrairement a ce qu'on constatait pour 1983. ce sont les travailleurs les moins instruits qui ont souvent les opinions les plus favorables au français. Il faudra procéder à d'autres analyses avant de se prononcer sur cette question.

Pour le moment. regardons plutôt le détail de ee type de relation en fonction du poste occupe el du sexe pour 1983 et 1990 , afin de comprendre davantage la situation terminologique et sociolinguistique de l'entreprise. de méme que le rôle véritable du sexe dans ce contexte.

\section{Le degré d'implication personnelle dans le processus de francisation de l'entreprise}

La figure 4 présente un double ensemble de données pour 198.3 et 1990) (groupe A). Les résultats sur le choix du français pour les 10 termes techniques testés en fonction du poste occupé sont repris et présentés en contraste avec les données concernant la perception que les travailleurs ont de se sentir. ou non, impliqués dans le processus de francisation de leur entreprise.

Il ressort que. tout comme le comportement terminologique déclaré. la perception concernant l'implication personnelle dans le processus de francisation de l'entreprise est en corrélation avec le poste. Et. encore une fois, on peut distinguer, en 1983. deux grandes catégories de travailleurs. Les cadres, gestionnaires et autres employés de hureau (à gauche de la figure) déclarent généralement une plus grande implication personnelle dans le processus de francisation de leur entreprise que les travailleurs cols bleus (à droite de la ligure). Les cadres. gestionnaires et représentants ont l'indice d'implication le plus élevé: 71.3: les secrélaires et autres employés de bureau ont. pour leur part. le troisième indice le plus élevé: 62.3. Les détenteurs de postes de supervision ont. éux aussi. en 1983. un indice d'implication personnelle élevé (6.3.4), qui contraste avec leur indice de francisattion terminologique, plutôt has. el qui s'apparente à ceux des travailleurs cols bleus, comme on l'a vu plus haut. Ils constituent done un groupe tampon qui participe aux tendances des deux groupes. Less mécaniciens, pour leur part, se situent à l'opposé du continuum, avec l'indice le plus has: 43.8 .

Toute proportion gardée, el malgré une baisse généralisée des indices, cette tendance se maintient en $1990^{2}=1$. Les cadres, gestionnaires et représentants obtiennent encore l'indice le plus élevé: 66.7, alors que lés mécaniciens ont le deuxième indice le plus bas: 46.1. Celte fois-ci. les répondants exerçant des fonctions de supervision font nettement partie du deuxième groupe de travailleurs composé principalement de cols bleus. Mais, et voilà qui est surprenant, les secrétaires et les autres employés de hureau. dont lindice de francisation est élevé tant en 198.3 qu en 1990. ont l'indice d'implication le plus bas: 43.7. 


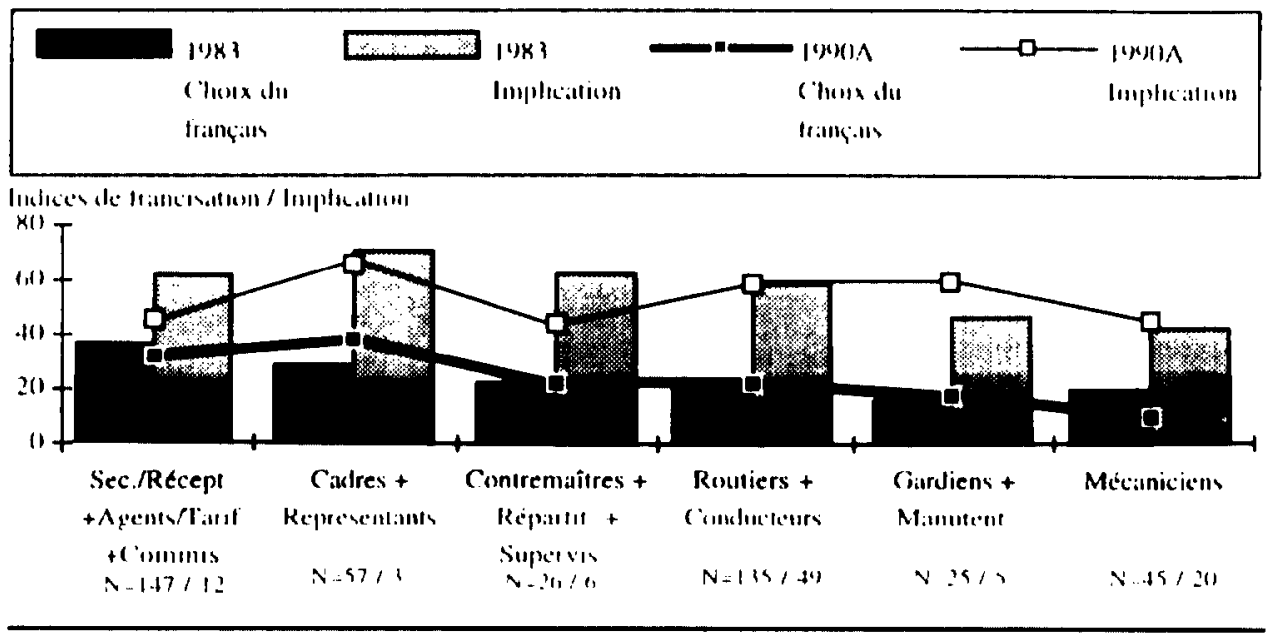

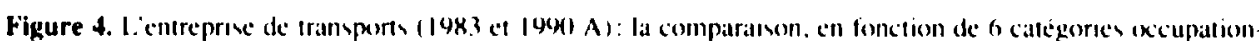

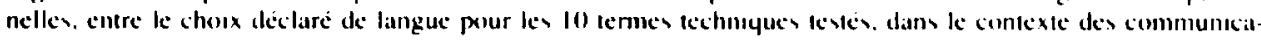
uons orales, el le degré demplication personnelle des travalleurs dans le precessus de francinateon de leur entreprive.

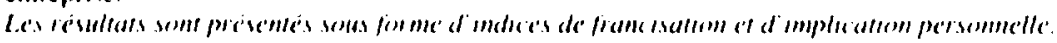

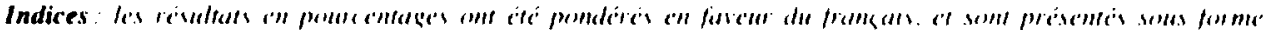

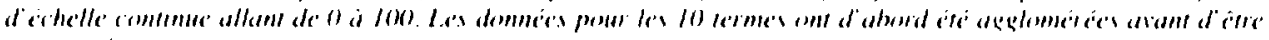

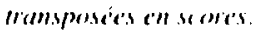

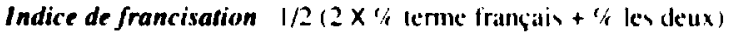

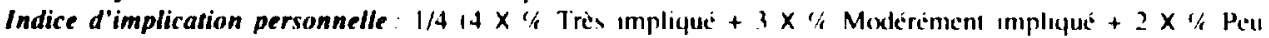
implique + 1/ Tre's peu impliques)

Test du khi carré (pour 1983): povte el implocalion personnelle: $P=<0,0,5: P=0,(x)$

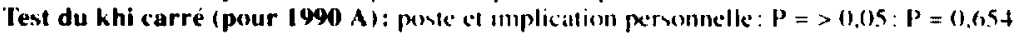

Seuls vom inclus dans le calcul ceux qui se sont prononces sur cetle question.

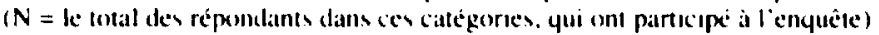

Comment interpréter cela? Et surtout. en quoi cette opinion a-t-elle un lien avec le comportement terminologique déclaré? Examinons tout d'abord ce dernier point. Si l'on regarde attentivement la figure 4 . On constate que, tant en 198.3 qu'en 1990). la courbe qui représente le choix du français pour les termes techniques est relativement parallèle à celle qui représente la perception des répondants par rapport à leur degré d’implication personnelle dans le processus de francisation. On en conclut que les deux variables. d'opinion et de comportement terminologique déclaré, semblent liées, elles aussi. Ce qui revient à dire que, dans un sens, la perception que les répondants ont de leur implication personnelle est un bon indice de leur degré d'utilisation du français. En d'autres mots, il semblerait que lon puisse dire que, plus un travailleur se sent concerne par la francisation de son entreprise. plus il y a de chances yu il choisisse plus souvent le français pour les termes techniques testés, et. vice versa. Cependant. même si ces deux types de variables (comportement terminologique déclaré et perception d'implication) sont en corrélation statistique avec le poste (pour 1983), il n'en reste pas moins qu'elles ne semblent pas entretenir des relations de même niveau. Ainsi. quel que soit le posle qu ils occupent. les répondants déclarent se sentir davantage impliqués dans le processus de francisation que ne le laisserait croire leur comportement terminologique déclaré. On voit cela en 
comparant les indices de francisation (pour les termes techniques) aux indices d'implication personnelle. Dans tous les cass. ce dernier indice est plus élevé. Il semblerait donc que les travailleurs ont une perception "exagérée" de leur utilisation déclarée du français pour les termes testés. Par contre. comme leur indice d'implication suit grossos mode la courbe de leur indice de francisation représentant leur choix de langue pour les termes, il semble clair que ces deux types de perceptions some liés?'. D'ailleurs, i l'on compare lat situation de 1983.3 avec celle de 1990) on se rend compte que lat courbe de l'implication personnelle suit encore célle du comportement déclaré. Ainsi, les secrétaires et autres employes de secrétariat. yui accusent une baisse au niveau du chosix du français pour les termes testés en 1990, accusent aussi une haisse au niveau du degré d'implication personnelle. Il en ess de même pour tous les groupes de travailleurs. que le changement se fasse vers une plus grande utilisation déclarée de termes français, ou l'inversé. Jen conclus qu un lien existe entre ces deux types de variables.

Essayons maintenant de comprendre le comportement des secrétaires et autres employés de bureau. Comment se fait-il qu'avec l'un des indices de francisation terminologique les plus élevés en 198.3 (36,8), et même encore en 1990) (32.9), leur degré d'implication personnelle ait chuté autant en 1990), pour passer de 62.3 a 4.3 .7 ?" He comment relier ceci à leur compontement terminologique déclaré?

\section{Le degré d'importance de l'utilisation d'une terminologie de langue française en milieu de travail et le degré de satisfaction de la situation terminologique}

Pour comprendre celat examinons d'autres opinions révélatrices d'attlitudes qui ont été testées en 1990, et qui sont lices aux attentes des travailleurs ${ }^{23}$. $\dot{A}$ la question demandant si. pour eux. il était important qu une terminologie de langue française soit employée au travail, une majorité de répondlants, de toutes catégories, ont répondu par l'affirmative, de sorte que le score obtenu par l'ensemble des travailleurs pour cette réponse est 70 . Lexamen de ces mêmes données, en fonction du poste excupé, confirme cette tendance générale, puisque les indices se situent entre 64.7 pour les mécaniciens et 76.1 pour les conducteurs et routiers. "est ce qu'on voit à la figure 5.

Il est intéressint de noter que les employés de secrétariat et de bureau ont un indice de 7.5 à la question relative a l'importance de l'utilisation d'une terminologie de langue française au travail. Il s'agit là du deuxième indice le plus élevé. On en conclut que cést une question importante pour eux.

Si l'on regarde maintenant les indices relatifs à la question portant sur le niveau de satisfaction de la situation terminologique dans leur entreprise. encore une fois. on constate que l'ensemble des répondants considère que la situation terminologique est satisfaisante. En effet, l'indice moyen pour l'ensemble des répondants, toutes catégories confondues. est de 73.8. Lexamen de ces mêmes données, mais en fonction du poste occupe, confirme celte tendance pour toutes les caltégories de postes sauf deux. Exception faite de cen deux postes. les indices sur cette yuestion vont de 7.5 pour les gardiens et manutentionnaires a 83.3 pour les cadres, gestionnaires et représentants. La situation est très différente pour les mécaniciens, qui ont un indice beaucoup plus bas, de 63.2. Mais ce sont les employés de secrétariat et de bureau qui ont l'indice le plus bas de tous: 57.1. II est évident que la situation terminologique de leur entreprise. en 1990, les déçoit. Pourtant, on a noté à plusieurs reprises que le groupe d'employés de secrétariat et de bureau est justement celui qui obtenait un des indices de francisation terminologique (pour les 10 termes testés) les plus élevés, autant en 1983 qu'en 1990). Cela veut dire qu'ils ont choisi plus souvent le français pour les 10 termes que la majorité de leurs collègues. De plus, on sait que ce même groupe avait un indice très élevé d'implication personnelle dauns la francisation de son entreprise en 1983. Mais on l'a noté, son indice 


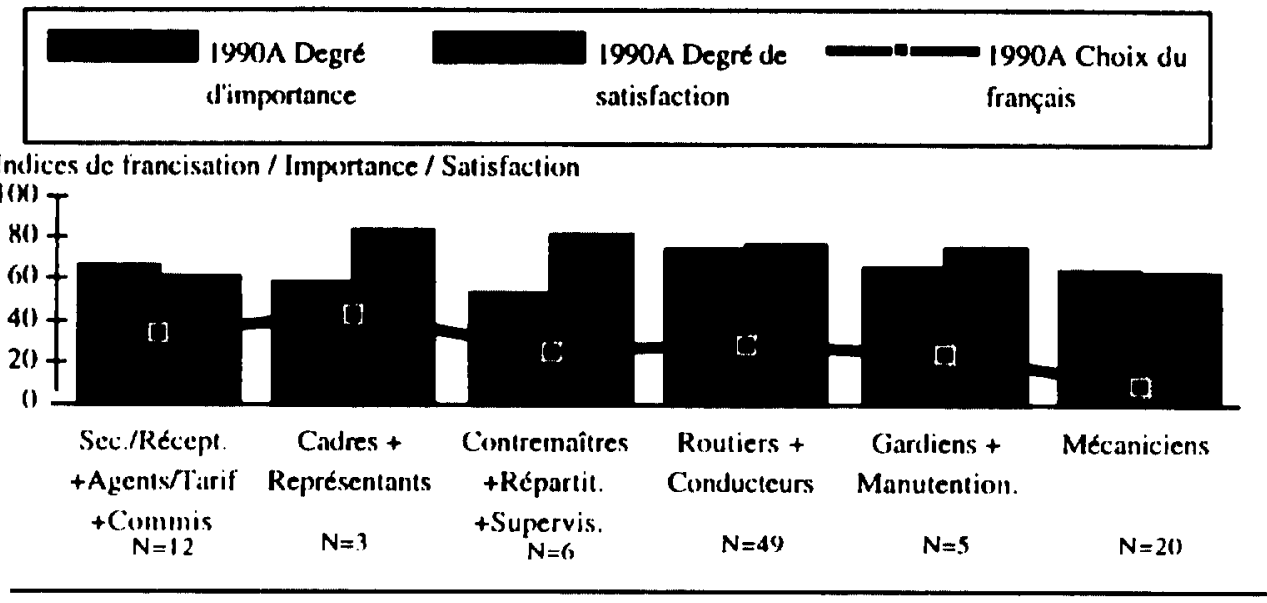

Figure 5. L'entreprisc de transpons $(\mid 490)$ A): la comparaison. en fonction de o catégories (xecupationnelles. entre le chovix declaré de langue pour les 10 tennes lechniques kesles, dans le contexte des communications

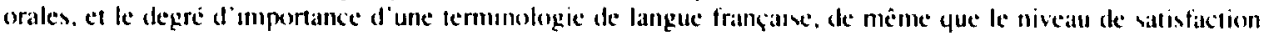
de la situation termmologeyue de l'entreprise.

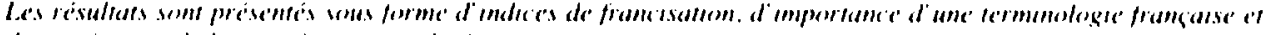

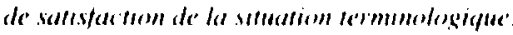

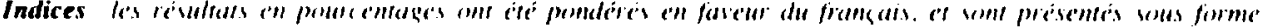

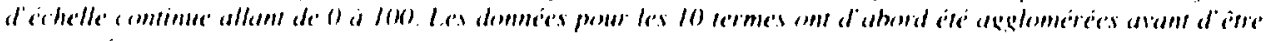
tramsposices en wiones

Indice de francisation $1 / 2$ (2X// lerme français + /4 lev deux )

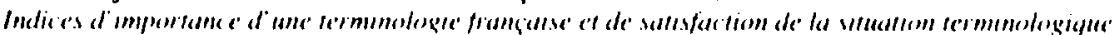

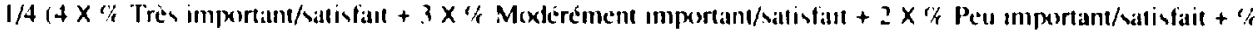
Tri-s peu important/satısfait)

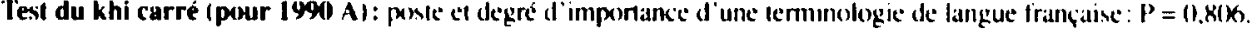
Test du khi carré (pour 1990 A): poste et niveau de satisfactule de la situation ferminulogicuc de l'entreprise $P=01.272$.

Seuls som inclus dans le calcul ceux qui se sont prenonces sur cette question.

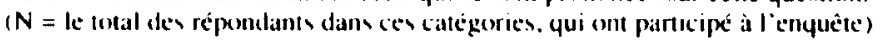

sur celle question avait chuté de 18.6 points en 1990), passant de 62.3 en 1983 à 43.7 en 1990. On en conclut que l'évolution terminologique qui s'est effectuée entre 1983 et 1990 ne correspond pas à ses allentes. Pourtant. c'est à peu près le contraire pour toutes les autres catégories de travailleurs. En fait. on a demandé à tous les travailleurs, en 1990. sils croyaient qu on utilisait davantage de termes lechniques de langue française dans leur entreprise qu avant (sur une périole de 5 ans). La majorité d'entre eux. toutes catégories confondues. ont répondu oui à celte question. les indices s'étendant entre 57.1 pour les superviseurs et 100$)$ pour les cadres, gestionnaires et représentants, ce dernier chiffre représentant. cela va sans dire. le plus haut taux de satisfaction possible. Encore une fois. cependant, les mécaniciens ont obtenu l’indice le plus bas: 25. Mais laissons de cóté la question du comportement des mécaniciens pour lequel les analyses actuelles ne permettent pas de foumir une explication. et examinons plutôt celui des secrétaires et autres employés de bureau. qui, cux aussi, ont un indice relativement bas (60)), qui les demarque des autres travailleurs cols blancs.

Que penser de leur comportement? D’où vient leur insalisfaction? Se pourrait-il qu encore une fois, lexplication soit liée à leurs fonctions? Comme on l'a déjà dit, less secrétaires, et. dans une moindre mesure, les autres employés de bureau, ont un rôle 
important au niveau de la francisation de l'entreprise. On a parlé de la pression exercée sur les secrétaires par la direction de l'entreprise pour qu elles assument la tâche de franciser les textes écrits et de donner un "visage" français a l'entreprise. On a supposé que cette fonction les a amenées à être davantage conscientes de la terminologie en langue française que les autres travailleurs. On peut même croire que, même si la campagne officielle de francisation terminologique n'est plus en cours depuis dix ans déjà, elles ont peut-être continué à jouer leur rôle d agentes de la francisation ${ }^{24}$. Dans l'étude de type qualitatif de 1993, nous avons justement posé cette question à certaines secrétaires qui nous ont répondu par l'aftirmative. De plus. il semblerait que même les secrétaires embauchées après 1983 aient reçu un mandat «implicitè de francisation. Si tel est le cas. on peut comprendre pourquoi les secrétaires semblent avoir des allentes plus élevées que les autres travailleurs et pourquoi, par le fait même, elles semblent décues des résultats.

Si l'on examine la question d'un autre point de vue, on se rend compte que, comme on l'a déjà dit, le groupe des secrétaires et autress employés de bureau comprend un grand nombre de femmes. Le corpus de 198.3 comprenait un total de 11.5 femmes. 10.3 d'entre elles occupaient des postes de secrétaires ou d'employées de bureau. Il est donc possible de croire que le phénomène d'insatisfaction des employés de secrétariat et de bureatu soit lié, en partie, tout au moins à des caractéristiques propres aux femmes. En 1990), le corpus ne comprend malheureusement que 12 femmes en tout. De ce nombre. 9 occupent des fonctions de secrétariat ou demployées de bureau. Le nombre est done trop petit pour se prononcer de façon définitive sur la question. Mais regardons tout de même les données déja présentées relatives au degré d'implication personnelle dans le processus de francisation de l'entreprise en fonction du sexe.

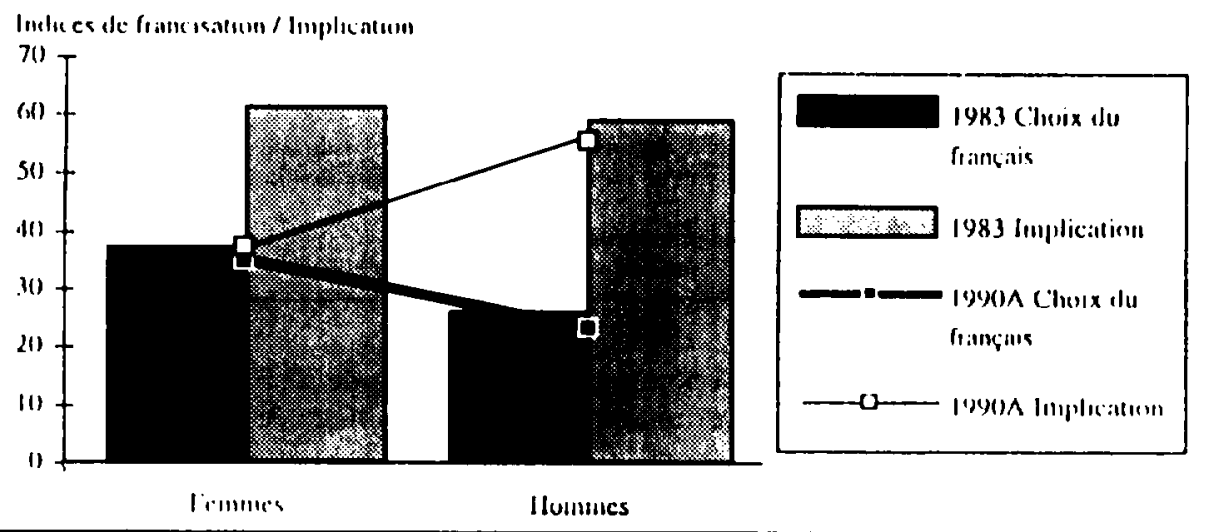

Figure 6. lientreprise de transport 11983 a 1990 A): la comparaison. en fonction du sexe. entre le chaix déclaré

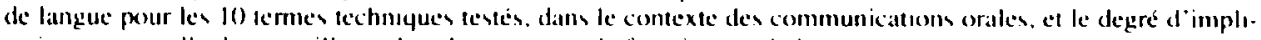
cation persennelle der travatilleurs datms le processus de francisatuon de leur entreprise.

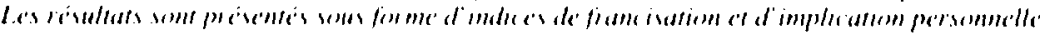

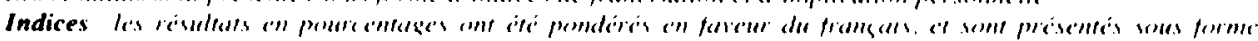

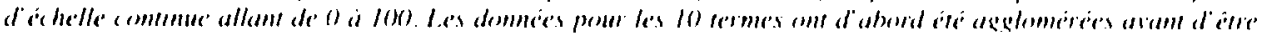
transposies an sertes

Indice de francisation $1 / 2(2 \times \%$ lerme frangals + "// lés deux $)$

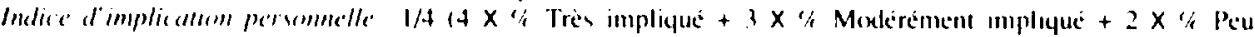
impliqué + 1/r Trios peu impliqué)

Test du khi carre (pour 198.3): wexe et implicalion perwonnelle: $P=>0.05: P=0.217$

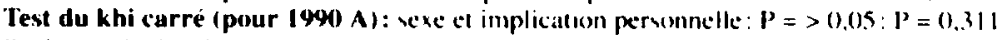

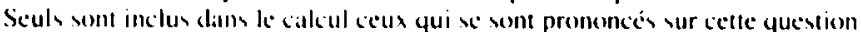

$(N=$ le total des repondants dans cen catégories, qui ont participe al lenquétel 
Les résultats présentés à la figure 6 révèlent que les variables présentées ne semblent pas entretenir le même type de relation en 1983 qu'en 1990. En 1983. on constate que. comme on l'a déjà vu. les femmes ont un indice de francisation terminologique plus élevé que les hommes. Cependant. les indices d'implication personnelle sont pratiquement les mêmes pour les hommes $(59,6)$, que pour les femmes $(61,2)$. On en conclut que le sexe ne semble pas exercer d'influence sur ce type d'opinion révélatrice d'attitude. Cela est corroboré par le test du khi carré qui ne permet pas d’établir de lien statistiquement signifieatif entre ces variables ( $P=>0,05)$. D'autres opinions du même genre, qu'on ne présentera pas ici. vont d’ailleurs dans le même sens. Il semblerait donc que, mème si le sexe semble être un ton indicaleur du comportement terminologique déclaré, il ne permette cependant pas d'élablir de liens avec certaines opinions révélatrices d’attitude. Cela justifie I'hypothèse présentée plus haut, selon laquelle le fait que les femmer déclarent un taux d'utilisation plus élevé de français pour les 10 termes testés est davantage lié aux fonctions qu'elles assument dans l'entreprise qu'au fait qu' elles soient des femmes. D'ailleurs, les analyses de régression effectuées sur le corpus de 198.3 confirment celte interprétation. Ceci ne veut pas dire, évidernment, que le sexe ne joue aucun rôle, mais simplement que d'autres facteurs, dont le poste, semblent exercer une intluence plus déterminante.

Si l'on regarde maintenant la figure 6 en fonction de la situation de 199(), on constate. comme on l'a déjà vu. que les femmes ont encore un indice de francisation terminologique plus élevé que les hommes. Pourtant, quand on examine les réponses à la question sur l'implication personnelle dans le processus de francisation. on constate que. contrairement à ce qu'on a déjà vu. cet indice ne suit pas une courbe similaire à celle du comportement terminologique déclaré en ce qui concerne les femmes. En fait. les femmes obtiennent un indice très bas d'implication personnelle (37.5), beaucoup plus bas, en fait. que leur indice de 1983 sur ce point (61.2). Quant aux hommes, leur indice d'implication personnelle est relativement élevé (55.7), presque autant qu en 1983 (59.6). Ces résultats confirment le fait que les femmes sont insatisfaites de la situation. Et. comme on sait que presque toutes les femmes occupent. en 1990, des postes de secrétariat ou demployées de bureau, on comprend davantage les résultats présentés en fonction du poste occupé. Mème si. en 1990 comme en 1983. les analyses statistiques $n$ ont pas établi de lien entre les opinions de ce genre et le sexe, la réaction des femmes reste intéressante et mérite qu' on s'y attarde. Ainsi, quand on a demandé aux répondants de 1990 s'ils croyaient que la situation terminologique de leur entreprise avait évolué vers un plus grand usage de termes lechniques de langue française au cours des 5 dernières années, les hommes ont obtenu un indice de 75.7 sur ce point. alors que les femmes en ont obtenu un de 55.6.

Cela me porte à croire qu il y a effectivement eu un changement entre 1983 et 1990). pas seulement en ce qui concerne le comportement terminologique déclaré, mais aussi el surtoul. peut-être, au niveau des opinions el comportements révélateurs d'attitudes. Même si les analyses statistiques sont loin dêtre complétées, il ressort que la majorité des répondants de 1990 (groupe A) semblent satisfaits de la situation terminologique et linguistique de leur entreprise. Mais certains groupes de travailleurs ne sont pas de cet avis. Ce sont les secrétaires et autres employés de bureau, de même que les mécaniciens. Ils semblent déçus de la situation. ce qui semble les rendre moins motivés à participer à lopération de francisation de leur entreprise. Fi les données de 1990) semblent indiquer que l'explication à tout cela est liée aux attentes des travailleurs. Les analyses préliminaires qu'on vient d'amorcer suite à l'enquête sur le terrain effectuée en 1993 permettent de croire que ces interprétations sont valables. Il s'avère que. en effet. un bon nombre des travailleurs rencontrés sont d'avis que leur entreprise sest francisée. La plupart d'entre eux semblent d'avis que leur milieu de travail est français. Beaucoup croient qu'il l'a toujours été. Quant à la situation terminologique, il semble clair qu elle paraît 
satisfaisante dans l'ensemble. exception faite de quelques-uns des 10 termes testés en 1983 et en 1990 qui, comme on l'a déjà dit, semblent être considérés comme des exceptions. Sur celte question des termes techniques. l'observation qu'on a fait sur place, tout comme les données de 1993, démontrent. en effet, qu un grand nombre des termes utilisés danss cette entreprise sont des termes français.

\section{CONCI.USION}

En conclusion, qu'est-ce qu'on peut retenir de l'analyse contrasté de la situation de 198.3 et de 1990"? II semble qu'en fait, on peut maintenant répondre à quelques-unes des questions posées au départ. Ainsi. cette étude a permis d"identifier certaines variables sociodémographiquess, occupationnelles. de même que certains types d'opinions révêlittrices d'altiludes qui semblent effectivement peser sur le comportement terminologique déclaré. On a aussi pu constater combien il fallait être prudent dams l'interprétation du comportement des travailleurs. Ainsi. l'analyse des données en fonction du sexe et du poste a permis de mettre at jour le fatit que le comportement est souvent fonction du rôle social des locuteurs. Cela a aussi mis en évidence le fait que le changement planifié n'obéit pass toujours aux mêmes règles que le changement naturel. De même on a aussi pu dégager. en partie du moins, la dynamique qui joue entre les variables sociodémographiques et occupationnelles el certaines perceptions ou opinions. Même si l'analyse débouche parfois sur des points d'interrogation. il reste néanmoins que les tendances générales dégagées paraissent valables et justifiées. Elles constituent autant de pistes de recherches qui devraient mener. Iorsque les analyses statistiques seront complétées, à une meilleure compréhension de la dynamique sociolinguistique qui sous-tend, d'une part. le choix des langues pour la terminologie, et d autre part le changement terminologique et sociolinguistique en milieu de travail. Une chose semble certaine: on ne peut examiner ces deux questions sans tenir compte des opinions, perceptions ou comportements révélateurs d'attitudes. et des liens complexes qu elles entretiennent avec les autres facteurs présentés ici.

Quant à savoir si le comportement terminologique déclaré peut être considéré comme le reflet du comportement terminologique réel. les données de létude sur le terrain (19933) semblent indiquer que, en 1993, comme en 198.3 et en 1990, kes 10 termes étudiés sont encore souvent utilisés en anglais. Dans ce sens, le comportement déclaré par les répondants de 198.3 et 1990 reflète celle situation el semble valable pour ces termes précis. Par contre, l'étude de 1993 a permis de constater qu un grand nombre d'autres termes sont utilisés en français, de sorte que. globatement, il est possible que l'examen de la situation terminologique réelle de l'entreprise s'avère davantage favorable au français que ce qui se dégage des données de 1983 et 1990.

Quoi qu'il en soit, une chose semble certaine: l'entreprise constitue vraiment une sous-communauté formée de différents groupes de travailleurs qui ont développé des comportements et des opinions conformes à leur groupe. Les analyses de 1983 et de 1990 le démontrent clairement. Dans ce sens. il semble plausible de croire que même si le comportement terminologique déclaré ne reflète sans doute pas exactement le comportement réel, il représente sans doute le consensus social de la communauté que forme lentreprise.

\section{Notes}

1. Ce programme de recherche a tete entrepris en 198.3 alors que je travaillais comme chercheur à l'Office de la langue frampaise. Je remercie les autorites de l'office de la langue françaixe de mavoir autorisce a poursuivre mes recherches dans le cadre de mes fonctions de professeur-chersheur à l'l lniversite du Quebec a

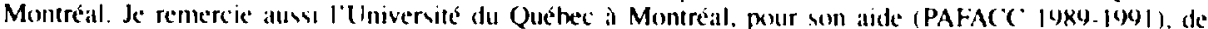

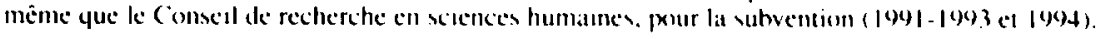


2. Organisme chargé de l"applicution de la "Charte de la langue françaisen ( "loi 101 ").

1. Pour une discussion sur les critères linguistiques et sociaux qui permettent d'établir une distinction entre les différentes categories d'emprunts lexicaux entre deux langues, voir, entre autres, Haugen 1950: Weinreich 1953: Poplack et al. 1988: Myers-Scotton 1992.

4. Nous adoptons ici le pount de vue de la sociolinguistique, qui prend comme ohjet d"elude non pas une communaté linguistique aidéale" caractériséte par l'utılisation d'une langue "homogente" (Chomsky 196.5 : 3-4). mais hien une acommunauté linguistique réelle" (L.yons 1970: 326). (ependant, contrairement it Lyons, yui adopte comme critere de base un critère linguisicque pour détinir la communauté linguistique (Lyons 1470), nous nous rallions à uns apprexhe du type de celle decrite ci-dessus, qui tient comple des normes sociales d utilisation des langues dans son critere de base (Fishman 1971 et 1 ahov 1666 et 1472 a et 1972b: 1.58). Pour sa part. Fishman 1971: 43 1 considere qu' "une communaute linguistique existe des linstant ou tous les membres ont au moins en commun une seule variéte linguistique, ainsi que les mormes de son emptoi correctm. Voir Ciumpers (1989: 133-164) pour une discussion générale de cetle approche sociolinguistique

5. La loi oblige toutes les entreprises comptant 100 employes et plus a obtenir un acertifical de francisation" qui atteste que l'entreprise est en train de se franciser, ou que le niveau de français qu'on y utilise satisfait aux exigences de la loi

6. Pour Lahov (1972b: 28.5). ce seratt la classe moyenne supérieure («ap)efer middle (lass") qui serait a l'origine de nombreux changements. Parfois, tres rarement. semble-t-il. le changement proviendrait de lat classe supérieure (Fasold 199(): 229). Pour une discussion des questions liées aux critères sous-jacents a la détinition des classes sociales, vour, entre autres, (iuy $(1988)$.

7. Pour une synthese et une analyse de toutes ces questions, voir, entre autres. Chambers el Trudgill (1980: 67-10) \& 16.3-181), Fasold ( 1990 ): 22.3.244). Newmeyer ( I988) et Sankoff ( 1986).

8. 1.étude que je decris ce i tait partie d'un programme de recherche qui comprend deux stries d'etudes de cas. En plus de l'entreprise de transports, dent il est question $x, j$ a aussi entrepris une étude similaire en $19 x 6$. dans deux entreprises manufacturieres de la chaussure.

9. Il va sans dire yu"il sagit w d'études de cas yui ne visent pas la representativite statistique au niveau des entreprises québicoises. (cependant. compte tenu de la méthode échantillonnale (le questronnatre a slé distribue a toute la population de l'entreprise) et de la taille des échantillom, surtout pour 19K.3. 10ut porte à crotre que les donnés constifuent des indices valables de la situation rélle.

10. $96.2 \%$ des travailleurs de 198.3 e1 $9.9 .9 \%$ de ceux de 1990 declarent avoir le français comme langue première. Il en va relativement de même pour ce qui est de la langue diusage déclarée.

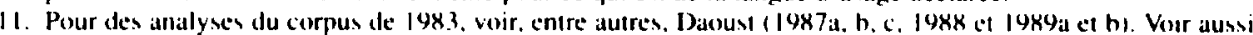

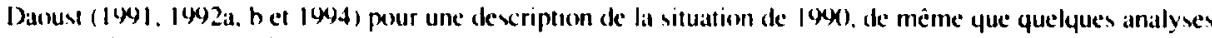
comparatives prelimmatires entre la situation de 198.3 et celle de loy).

12. Mème si la coxlification dev donnes de 199,3 vient à peine de commencer. Il semble yue, du moins pour ce qui est des entrevues semj-dirigeses, on trouve entre $3(x)$ et $5(x)$ excurrences de temes lechniques pour chac un des repondants. Quant au nombre d'occursences de lemes différents prononces par les répondants. mentionnons que dans deux entrevues prises au hasard. nous avom relevé press de I 80 termes differents.

1.3. Jusqu'à maintenant. chacun des termes est clasé selon une yuarantaine de var lables tenant compte, entre autres. du degré de formalité de la situation dans layuelle a est utiliste de differents noveaux de lechnicite. de différentes caractéristicfues languistiques sur la prononesation. la langue d"origine, le champ synonymauce et sémanicues, etci

14. Je remercie Monsieur David Sanhoft du centre de recherches mathématiques de I'Iniverslle de Montreal. d'avesir eflectue une bonne partie des analyses statisliques du corpun de $19 \times 3$. et de m avolr conseillee quant a l'interpretation et a l'analyse de certaines donneses. Bien entendu. je demeure stule responsable de" erreurs qu auraient pu se glisser relativement at la demarche generale el a l'interprétation de l'ensemble des résultats.

15. Rappelons qué, comme nous l'avons dejà mentionné, aucune analysce quantitative n a cencore ćté laste sur les donnes de 149,3 .

16. Le canal de communication. c'est-à-dire l'utulisation de l'oral ou de l'écrte pése ansi sur le choux de langue pour les ménes lo termes lechniques testes (Dinous 1984a).

17. Le lest du hhi sarre a cté effectue sur les donnés avant qu elles ne soient transponces sous forme d indices. II en est de mème pour les autres résultats présentés ci-dessous.

18. Dautres analyses statisliques vont justement prévues. De plus, on prevesil d'harmoniser les 3 corpus de

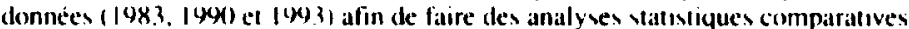

19. II en est de mème pour le corpus de $l(x)$, où. malheureusement le trop pertit nombre de secretaires (N $=3$ ) IIe promet pas de vérifict l'hypothese présenté ci-dessus.

20. Malheureusement, dans nolre cchantillon de $190 x$ (groupe A). les postes de gestionnaires de lous niveaux et de cols hlancs en général soni sus-representés, a cause, non pas uniquement de la taille de notre echantallon, malls surtout parce que peu de travailleurs qui oceupaient ces postes en 198.3 étatent encore priscons 
dans l'entreprise en 1990). En fait. non seulement il y a plus de mobilité au niveau de ces enployés. de sorte que bon nombre d'entre eux ont quitté. mais de plus. les changements organisationnels ont vurtoul touche ces categories d'employes, particulierement les gestionnatres de plus hatut niveatu, de sorte que très peu d'entre eux somt encore dans l'entreprise. ("est pourquai il faut ëtre prudent dans l'interprétation de certaines analyses statistiques.

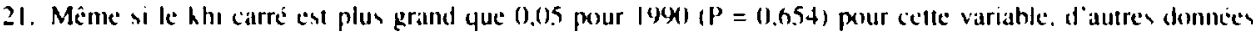
portent a croirc que le posti joue un rôle important.

22. Plus encore, le degré même décart entre les perceptions deimplication personnelle et le comporlement terminologique déclarés est fonctuon du poste excupe. Ainsi, les cadres. gestionnaires ed alutres cols blancs accusent un ecart moins imporlant que les travailleurs cols bleus. (n en conclut que ces travailleurs ont une perception plus "réaliste" de leur comportement declare. (e schema vaut pour d"autres variables, cotmme

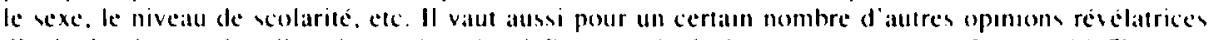

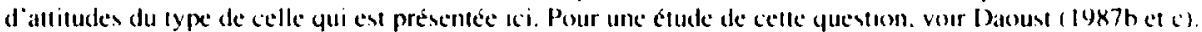

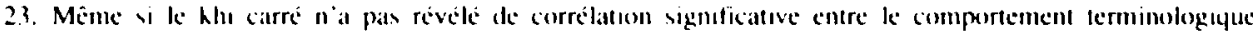
declare et les deux variables preventees dans la figure 5. tout porte a croire yue cela est dú a la taille de lechantillom. Datutes lests statistiques, plus adaptés à la petite laalle du corpus, seront effectues. Je presente

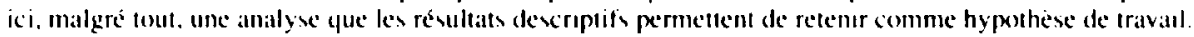

24. Sur ce point. il faut signaler que. lorsque la campagne de francesation a dibute en lok.3. allers yue je collectais les donnes. l'entreprise avalt entrepris un programme de senshilisation a la francisillon ce avatl contic at certains employes le mandat de franciser leur entourage. (és employes avanent méme un ture: ales agents 101 " (du nom de la (harte de la langue françalse, dite loi 101). Plusieurs secrélaires faisaient partic de ce groupe.

\section{RFFERENCWS}

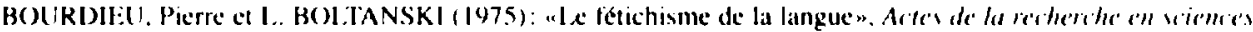
werales. n' $4, \mathrm{pp} .2-32$

BOURHIS, Richard Y. (198.3): "L anguage Attifudes and Self Reports of French-Fnglish l.anguage lisage In

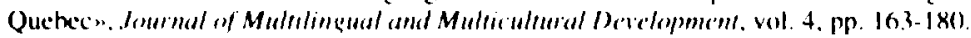

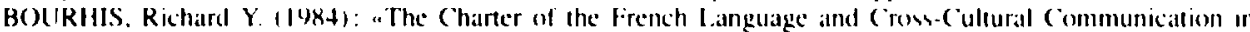

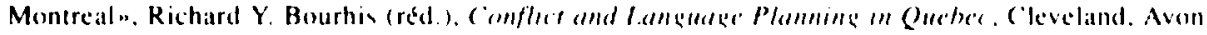
PP. 174.204

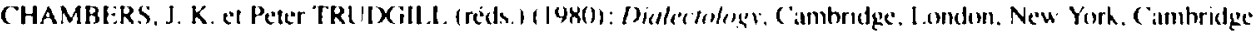
Iniversity Press, $21 \times p$

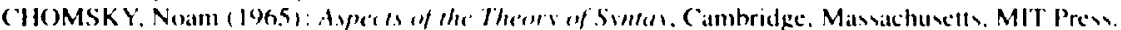

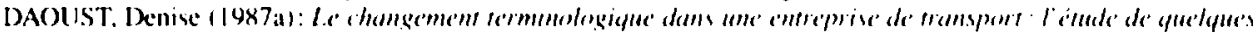

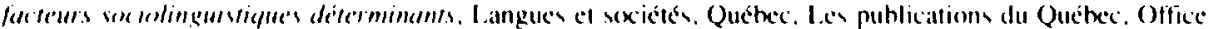
de la langue française, $149 \mathrm{p}$.

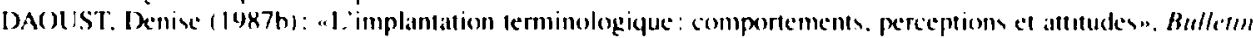

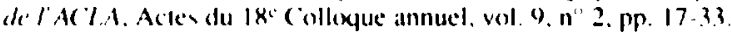

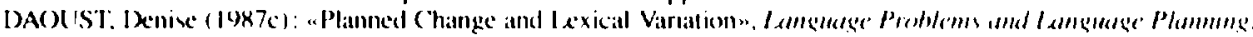
vol. 11. n' 2. pp. 148-165.

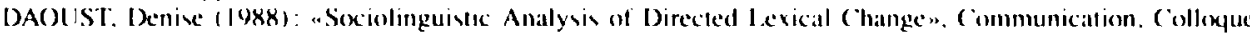
international sur lat variation linguistique. NWAVE-XVIll, liniversite de Montreal. ms.

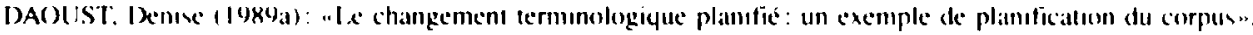

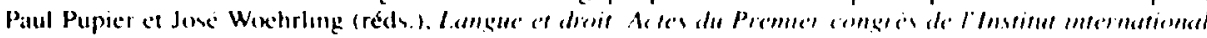

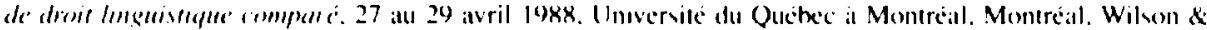
l atleur lice. pp. 615.641

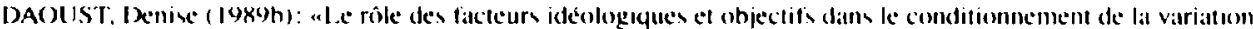

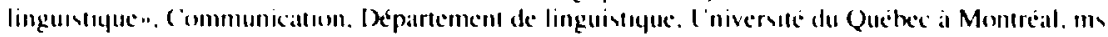

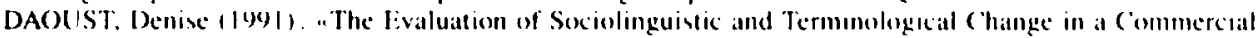

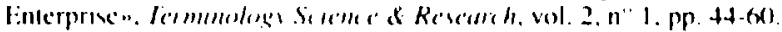

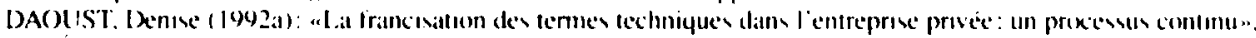

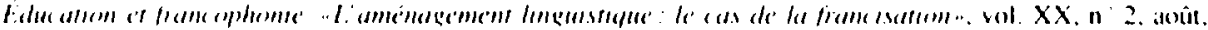
pp. 42.51

DAOUST. Demse (1942b): "Le rôle du poste comme tacteur de changement des habitudes termanologicues

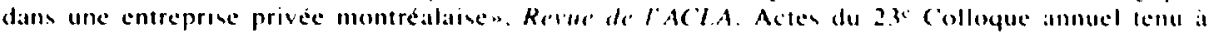

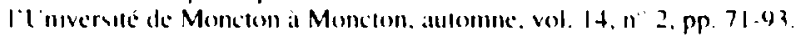

DAOUST. Denise 1 1994): "L importance de quelques opimoms et attitudes sur le comportement termimologeque

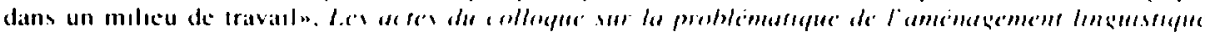

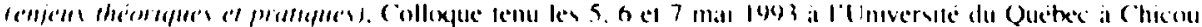


timı. (iouvernement du Québec. Office de la langue française et Unıversité du Québec a Chicoutimi. pp. 1.37-177

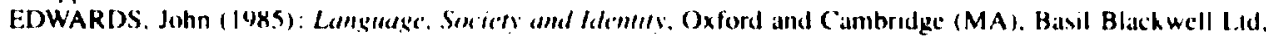
$24.5 \mathrm{p}$.

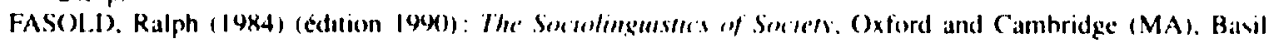
Blachwell l.td, 341 p.

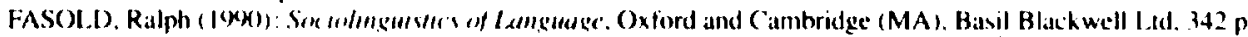

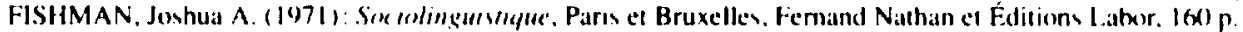

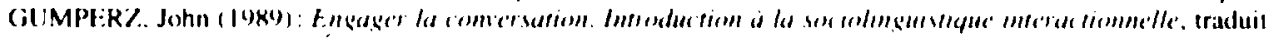

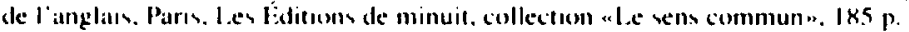

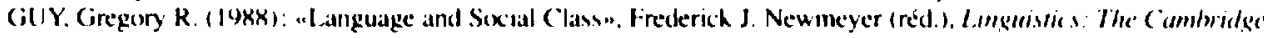

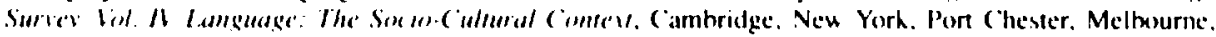
Sydney. Cambridge llmversity Prews, pp. 37-6.3.

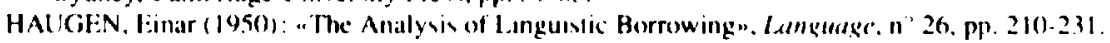

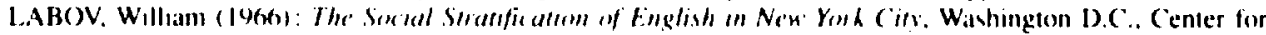
Applied linguistics.

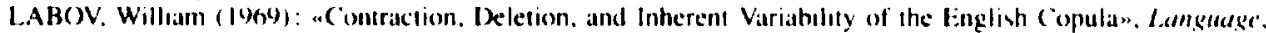
n" 45. pp. $715-762$

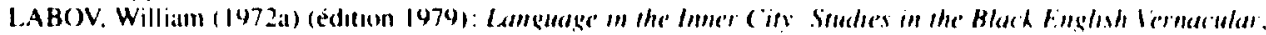
Philadelphic. I nnversity of Pennsylvania Press. $412 p$

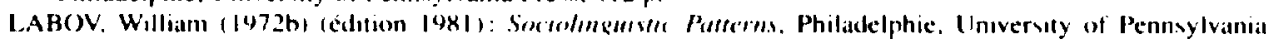
Press. $34.3 \mathrm{n}$

LABOV. William (|98|): “What Can Be Learned About Change in Progress Irom Synchronc Description?";

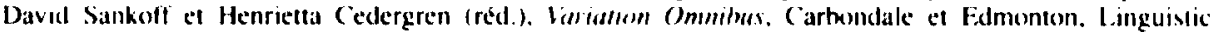
Research Inc.. pp. 177-199.

LABOV. Willam ( $\mid(x)$ ) :The Intersection of Sex and Soxial Class in the Course of linguistic (hange".

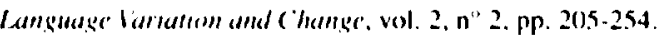

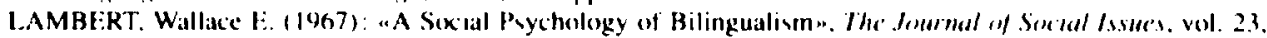
n" 2 , pp. $91-1($ K)

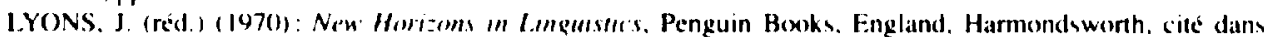

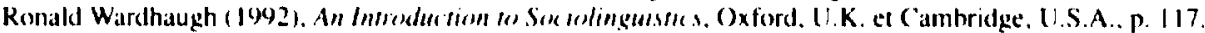

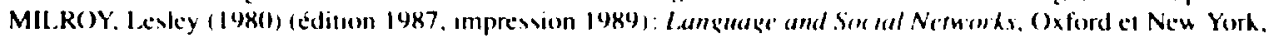
Basil Blackwell Inc., $2.32 \mathrm{p}$

MIL.ROY. Lesley (1987) (impression 149(1): Obscrimg \& Analyzing Natural Language. Oxford et New York. Basil Blackwell Inc.. 230 p.

MYERS-SCOTTON. Carol (1992): "Comparing Codeswikching and Borrowing". Jourmal of Mulmlingual amb

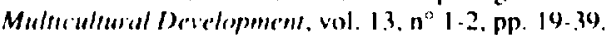

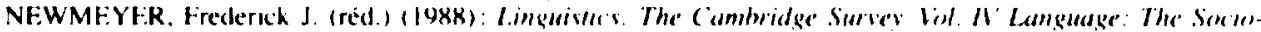
Cullural comlev. Cambridge. New York. Port Chester. Melbourne. Sydney. Cambridge Inniversity Press. $292 \mathrm{p}$.

P()PLACK. Shana. SANKOFF, David et (hristopher MILL.ER (I98X): "The Soxial Correlates and Linguistic

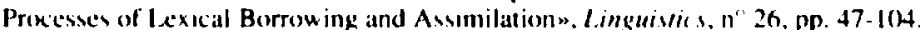

SANKOFF, David (red.) (1986): Drversm and Diacherm): (Current lasues in l.inguistic Theory. $n^{\prime \prime}$ 53. Amsterdam/ Philadelphia. John Benjamim. Publishing Company. $430 \mathrm{p}$.

SANKOFI: David et Suzanne LABER(iE: (1978): "The Linguistic Marhet and the Statistical Fxplanation of

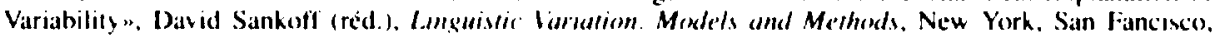
l.ondon, Academic P're's, pp. 234-250.

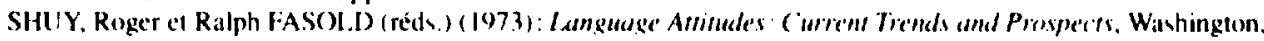
Georgetown Universily Prew. $201 \mathrm{p}$.

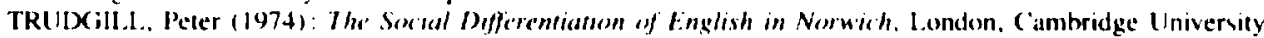

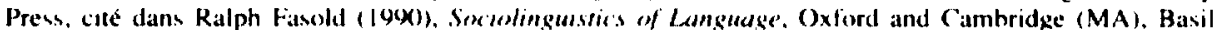
Blachwell L.dd. pp. 222-268

TRUIX ill.1. Peter (1979): :Standard and Non-Standard Dialects of English in the Unted Kingdom: Problem.

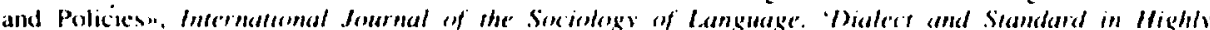

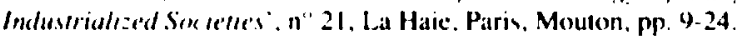

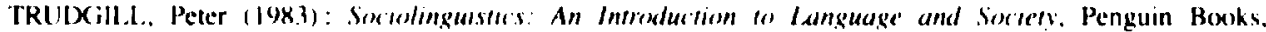
England, Llarmondsworth

WEINREICH. (Iricl (1953) (édition 1966): languages in Contact. Findings and Prohtems. La Haie el Paris. Mouton. $14 \times \mathrm{p}$.

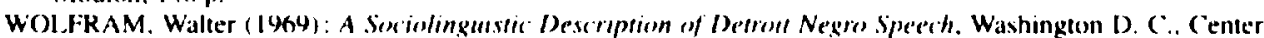
for Applied l. Inguistics. 